\title{
Turbulence decay in a supersonic boundary layer subjected to a transverse sonic jet
}

\begin{tabular}{|r|l|}
\hline Journal: & Journal of Fluid Mechanics \\
\hline Manuscript ID & JFM-18-S-1111 \\
\hline mss type: & JFM Papers \\
\hline Date Submitted by the Author: & 14-Aug-2018 \\
\hline Complete List of Authors: & $\begin{array}{l}\text { Sun, Mingbo; Science and Technology on Scramjet Lab } \\
\text { Liu, Yuan; National University of Defense Technology, Science and } \\
\text { Technology on Scramjet Lab } \\
\text { Hu, Zhiwei; University of Southampton, SES-Aero }\end{array}$ \\
\hline Keyword: & $\begin{array}{l}\text { High-speed flow < Aerodynamics, Compressible boundary layers < } \\
\text { Compressible Flows, Jets < Wakes/Jets }\end{array}$ \\
\hline \multicolumn{2}{|c}{} \\
\hline
\end{tabular}




\title{
Turbulence decay in a supersonic boundary layer subjected to a transverse sonic jet
}

\author{
Mingbo Sun ${ }^{1}$, Yuan Liu ${ }^{1}$, Zhiwei $\mathrm{Hu}^{2}$ \\ ${ }^{1}$ Science and Technology on Scramjet Laboratory, National University of Defense Technology, \\ Changsha, 410073, China
}

${ }^{2}$ Faculty of Engineering and the Environment, University of Southampton, Southampton, SO17

$1 \mathrm{BJ}, \mathrm{UK}$

\begin{abstract}
The turbulence status in a supersonic boundary layer subjected to a transverse sonic jet is studied by conducting direct numerical simulations. Turbulence statistics about two different jet-to-cross-flow momentum flux ratios $(J)$ of 2.3 and 5.5 based on the previous simulation (Sun M B, Hu Z W, J. Fluid Mech., 2018, 850:551-583) are given and compared with a flat plate boundary layer without jet $(J=0.0)$. The instantaneous and time-averaged flow features around the transverse jet in the supersonic boundary layer are analyzed. It is found that in the near-wall region, turbulence is suppressed significantly with increasing $J$ in the lateral boundary layer around the jet and the status retains in the downstream recovery region. The local boundary layer thickness decreases obviously in the lateral downstream of the jet. Analysis of the cross-flow streamlines reveals a double-expansion character in the vicinity of the jet, which involves the reattachment expansion related to the flow over the jet windward separation bubble and the jet lateral expansion related to the flow around the jet barrel shock. The double expansion leads to the turbulence decay in the jet lateral boundary layer and causes the slow recovery of the outer layer in the far-field boundary layer. A preliminary experiment based on nano-particle laser scattering technique is conducted and confirms the existence of the turbulence decay phenomenon.
\end{abstract}


Keywords: supersonic cross-flow; transverse jet; turbulent boundary layer; turbulence decay

\section{Introduction}

In scramjet engine combustors, fuel is injected normally through sonic jets from wall orifice into a supersonic cross-flow of air, which is a simple and efficient injection scheme for combustor design. A significant body of work has been summarized in the previous reviews (Karagozian (2010) and Mahesh (2013)). Many experimental investigations have been carried out to understand the mechanisms of the jet injected into a supersonic cross-flow, including detailed velocity (Santiago \& Dutton 1997) and wall-pressure measurements (Everett et al. 1998), penetration height (Portz \& Segal 2006) and time-resolved flow visualizations (Gruber et al. 1997; Sun et al. 2013) and analysis of mixing characteristics (Ben-Yakar, Mungal \& Hanson 2006; Gamba \& Mungal 2015) with nonreactive and combustible gaseous jets. These measurements showed the overall jet flow features and the dynamics of the jet shear layer and shocks along with intensive flow data that can be used to validate numerical predictions. Because of the difficulties in measuring the complex high-speed unsteady flow fields, experimental data are mainly obtained for certain transverse and longitudinal two-dimensional slices of the flow field.

A widely accepted flow topology of a transverse jet through an under-expanded sonic injection from a wall surface to a supersonic cross-flow is illustrated in figure 1 . The flow field involves complex three-dimensional (3D) unsteady shocks, separation, wall-bounded turbulence, vortical structures and their interactions (Dickmann \& Lu 2009). A barrel shock around the plume terminating in a Mach disk and a bow shock is generated ahead of the jet. This causes boundary-layer separation upstream of the injection and leads to the formation of a horseshoe vortex. A local leeward separation bubble and a secondary shock is presented. Recently Liang et al. 
(2018) identified that a reflected shock deflects the collision shock and the collision shock intersects with the barrel shock, as shown in figure 1(b), which clarifies the knowledge about the shock wave structures in the jet wake. The previous study had also well revealed the jet vortices resulting from the sonic transverse jet injected into the supersonic cross-flow. The formation of a major counter-rotating vortex pair (CVP) and horseshoe vortices has also been well explained. Our research work on jet interaction with supersonic cross-flow (JISC) clarified the upper trailing CVP (Sun \& Hu, 2018b) and surface lower trailing CVP (Sun \& Hu, 2018a).

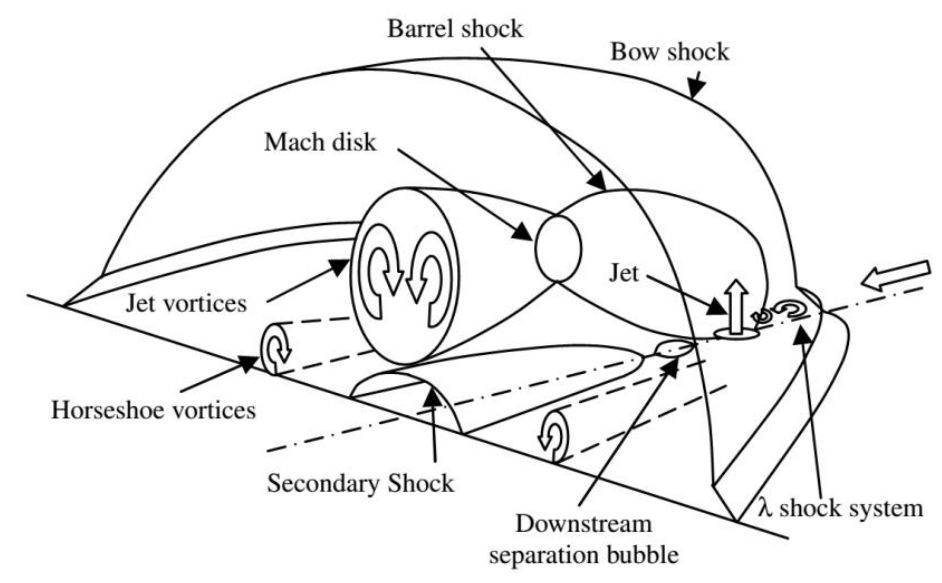

(a) The 3D view of near-field mean-flow structure (Dickmann \& Lu 2009)

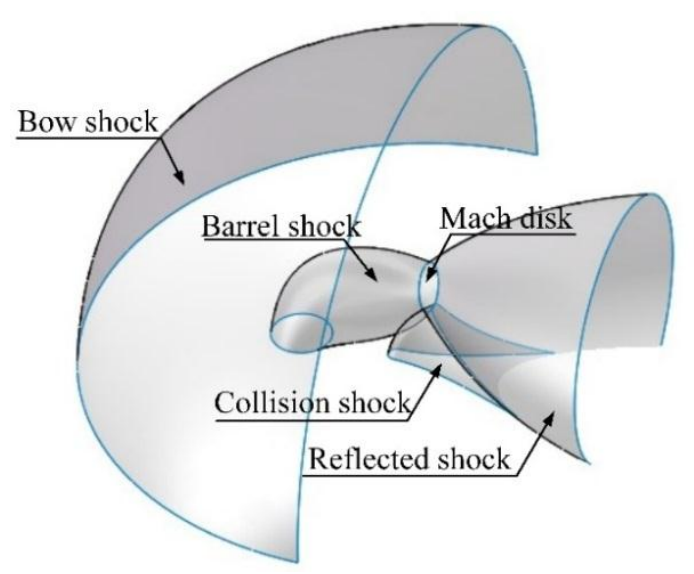

(b) The 3D view of near-field shock structure (Liang et al. 2018)

FIGURE 1. 3D view of near-field mean flow structure of a sonic jet into a supersonic cross-flow Recently a number of detailed numerical simulations have been conducted to obtain the 
detailed flow field and to analyze the behavior of coherent structures. Kawai \& Lele (2010) and Rana et al. (2011) performed large eddy simulations (LES) of sonic jets with $J=1.7$ in a supersonic stream and their results demonstrated Kelvin-Helmholtz $(\mathrm{K}-\mathrm{H})$ instabilities in the interface of the jet shear layer confronted with the main air-stream. Won et al. (2010) used detached-eddy simulation (DES) to reveal vortex evolvement under the jet conditions of Ben-Yakar's experiment (Ben-Yakar, Mungal \& Hanson 2006). Chai, Iyer \& Mahesh (2015) conducted LES on jets in a supersonic cross-flow. Though the flow field of those above simulations in the boundary layer was demonstrated, no details were discussed on the effects of jet interaction on the supersonic boundary layer. And the turbulence evolvement in the boundary layer of the jet wakes has not been well revealed in the literature either. As is well known, turbulence status in the boundary layer is important for the skin friction and heat transfer, therefore it is useful to know any change of the turbulence in the boundary layer around the jet. Previous experiments have not demonstrated this topic, while RANS or LES requires modeling of all or the small-scales of turbulence. In the present work, DNS is used to obtain detailed information on the turbulence around the jet in the boundary layer based on the previous simulation (Sun \& Hu, 2018b). The significance of this investigation lies in the identification of turbulence status in the supersonic boundary layer around the transverse jet, which has been overlooked by previous studies.

This paper is organized as follows. The computational grid, the algorithm, the simulation conditions and the validation are discussed in Section 2. A description of the instantaneous boundary layer flow field is presented in Section 3.1. Section 3.2 gives the mean flow field and properties of turbulent kinetic energy and root-mean-square (RMS) velocity distributions. Analysis of streamlines which illustrate flow field characteristics related to the change of local 
turbulence is presented in Section 4. A preliminary experimental study is discussed in Section 5 and the main description is given in Appendix A-B since the experiment validation is qualitative. A summary is given in Section 6.

\section{Flow conditions and numerical setup}

\subsection{Numerical simulation and inflow condition}

All simulations in this paper solve the 3D unsteady compressible Navier-Stokes equations directly without any modeling, using an in-house DNS code. The code has been developed and applied to studies of instability, transition and turbulence in high-speed flows (Sandham et al. 2014; Sandham 2016). Here we provide a brief description of the main features of the code and explain the simulation conditions for the present study. The detailed governing equations of continuity, momentum, total energy for 3D flow field can be found in Touber (2010) thus are not repeated here.

The air inflow parameters (shown in Table I) are set in accordance with the $M a=2.7$ experiments of Sun et al. (2013) with stagnation pressure $P_{0}=101,325 \mathrm{~Pa}$ and stagnation temperature $T_{0}=300 \mathrm{~K}$. The bottom wall inflow $99 \%$ boundary-layer thickness, which is the same for all simulations, is estimated to be $\delta_{i}=5.12 \mathrm{~mm}$, with the compressible (including density variations) boundary-layer displacement $\delta_{i}{ }^{*}=1.75 \mathrm{~mm}$ and momentum thicknesses $\theta=0.38 \mathrm{~mm}$ and corresponding Reynolds numbers $R e_{\delta^{*}}=15,367, R e_{\theta}=3,337$ respectively. A sketch of the computational domain is shown in the previous papers (Sun \& Hu 2018a; Sun \& Hu 2018b) and for brevity omitted here.

Two momentum flux ratios $J=2.3$ and $J=5.5$ of air injection are modeled. Both cases lead to 
a sonic jet with stagnation temperature $T_{0 \mathrm{i}}=300 \mathrm{~K}$. The jet properties are shown in Table II. Since the turbulent levels originating from the jet orifice in the experiments are not known, a uniform profile across the jet orifice is implemented without any disturbance. It might be argued that turbulence would not be thoroughly resolved in the jet; however, it is believed that the overall behavior of coherent structures in jet and boundary layer will not be affected by this assumption (Sun \& Hu 2018a; Sun \& Hu 2018b).

TABLE I. Air flow conditions for the simulations, including the dimensional boundary-layer (BL) thicknesses and Reynolds number at the inflow

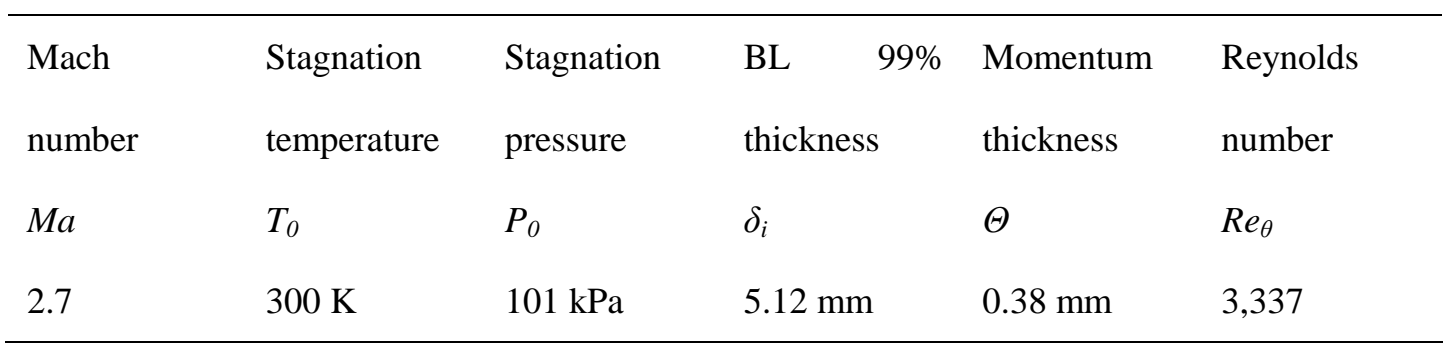

TABLE II. Jet conditions for the simulations

\begin{tabular}{|c|c|c|c|c|}
\hline Jet-to-crossflow & Mach number & orifice & Stagnation & Stagnation \\
\hline momentum flux ratio & & diameter & temperature & pressure \\
\hline$J$ & $M a_{j}$ & $D$ & $T_{0 j}$ & $P_{0 j}$ \\
\hline 0 & Non & Non & Non & Non \\
\hline 2.3 & 1.0 & $2 \mathrm{~mm}$ & $300 \mathrm{~K}$ & $138 \mathrm{kPa}$ \\
\hline 5.5 & 1.0 & $2 \mathrm{~mm}$ & $300 \mathrm{~K}$ & $330 \mathrm{kPa}$ \\
\hline
\end{tabular}

\subsection{Computational domain and boundary conditions}

The use of a digital filter to generate the inflow boundary condition significantly reduces the length required for boundary-layer development (Xie \& Castro 2008), but nevertheless there 
should be enough distance provided in the simulation to allow the boundary layers to adjust to an equilibrium state upstream of the jet. Wang et al. (2015) found that a distance of 12 times the inflow boundary-layer thickness is enough to obtain realistic turbulent mean and RMS profiles from the digital filter inflow generator used. Accordingly, the initial length is set to be $L_{i}=12.7 \delta_{i} \approx 65 \mathrm{~mm}$ for a fully-developed turbulence generation. The jet mixing length is set to $L_{m}=90 \mathrm{~mm}$ including the near-field (denoted as JISC section1) with $10 \mathrm{~mm}$ upstream and $40 \mathrm{~mm}$ downstream of the jet and the far-field (denoted as JISC section2) with $40 \mathrm{~mm}$ downstream of JISC section1. Therefore, the total length of the physical domain is $L_{x}=155 \mathrm{~mm}$. The wall-normal extent of the domain is $18 \mathrm{~mm}$, and a span of $14 \mathrm{~mm}$ is used. The jet is centred $75 \mathrm{~mm}$ downstream of the inlet with an orifice diameter of $D=2 \mathrm{~mm}$. The grid used in the current simulation is kept the same with the setup in our previous work (Sun \& Hu 2018a; Sun \& Hu 2018b). As analyzed, the grid setup ensures a fine DNS resolution in the near-wall region and a quasi-DNS (QDNS) resolution in the jet and the mainstream due to the unknown information about the smallest scales in the jet plume, as denoted by Sandham, Johnstone \& Jacobs (2017).

Large buffer layers with non-reflective outside boundaries are introduced with lengths of 15 $\mathrm{mm}, 11 \mathrm{~mm}$ and $12 \mathrm{~mm}$ at the outlet, on both the sides and the upper boundaries, respectively. The grid is smoothly stretched in the buffer layers towards the boundaries. The buffer layers, with coarsened mesh, help to damp fluctuations and minimize any possible reflections from the boundaries. Non-slip and adiabatic boundary conditions are enforced on the bottom wall. An integrated characteristic boundary condition (Thompson 1987) is applied at the inflow, and an outflow condition with an integrated characteristic scheme is applied to the spanwise, top and outflow boundaries, which are located on the edge of the buffer layers. The jet orifice is defined 
directly in the surface wall uniform grid and set to a sonic condition with $J=2.3$ and $J=5.5$, respectively.

\subsection{Numerical methods and validation}

This in-house code (Sandham, Li \& Yee 2002) uses a fourth-order central difference scheme for the spatial derivatives and a third-order explicit Runge-Kutta scheme for time advancement. The code makes use of an entropy splitting of the Euler terms and the Laplacian formulation of the viscous terms to improve the stability of the non-dissipative central scheme. To better resolve the steep gradient in flow field around the injected jet, we developed a hybrid scheme which combines the entropy splitting central scheme with a central-upwind WENO-CU4 scheme and is used in the areas with high gradients (Sun \& Hu 2018b).

The digital filter inflow generation method of Xie and Castro (2008) is used to provide inflow boundary conditions to generate a more realistic turbulent boundary layer, from pre-defined mean and root-mean-square (RMS) profiles. The mean inflow profiles are generated from similarity solution of compressible boundary layer using the same approach as Wang et al (2015). The inflow RMS values are taken from the DNS results of Schlatter \& Orlu (2010) for a similar Reynolds number.

Turbulent boundary-layer mean profiles and distributions of RMS values at $x / D=-7.5$ (corresponding to the position at $60 \mathrm{~mm}$ downstream of the inlet) have been evaluated, giving a good agreement with the existing data (Sun \& Hu 2018a; Sun \& Hu 2018b). The approximate penetration boundary based on a concentration identification was obtained and compared with the empirical correlation. It was found that the current penetration result agrees well with previous experimental correlations for both $J=5.5$ and $J=2.3$ (Sun \& Hu 2018a). The jet trajectory of $J=5.5$ 
and $J=2.3$ was extracted from the streamline originating from the centre of the jet exit on the time-averaged symmetry plane. It was compared with the Schetz \& Billig (1966)'s prediction and showed reasonably good agreement (Sun \& Hu 2018a). The above comparison validated the approach and the current simulation.

\section{Numerical results and analysis}

All simulations were performed on Archer, UK National Supercomputer. Each simulation requires approximately 540,000 processor core hours for flow development and collecting statistics over 100 non-dimensional time units $\left(D / U_{\infty}\right)$.

\subsection{Instantaneous flow structures}

Contours of typical instantaneous density fields for the supersonic boundary layer without and with the jet injection are compared in figure 2. Figure 2(a) reveals detailed structures of the boundary layer and unsteady jet penetration. The injected jet introduces a bow shock, a barrel shock and separation bubbles in the boundary layer, which are well identified in the simulations. The upstream separation shock is not as strong as the other shocks. Upstream of the jet, a recirculation region is observed. The vortical structures around the jet break down to form the well-developed small turbulent eddies downstream. Analysis of the jet structure can be found in previous literatures, therefore is not detailed here. The present study focuses on the boundary layer developments downstream of the jet. It is found that the jet interacts with the upcoming boundary layer intensively and the interaction is still strong in the downstream region in the centerline plane $(z / D=0)$. Figure $2(b)$ gives the flow field on the plane of $z / D=3.0$ for all cases. From this side-view, the vortices in the boundary layer of $J=5.5$ and $J=2.3$ case are both observed to be suppressed, 
while the phenomenon is more obvious for $J=5.5$. The vortical structures are attenuated, and start to re-organize after a long distance (denoted as 'recovery' in this paper) in the region downstream of the jet, indicated by the red arrows shown in figure $2(b)$. For $J=5.5$, this recovery distance is longer and part of the boundary layer even approaches to the laminar state, which means that higher $J$ leads to a more intensive vortex decay and even a laminarization downstream of the jet.

0.050 .250 .450 .650 .851 .051 .251 .45
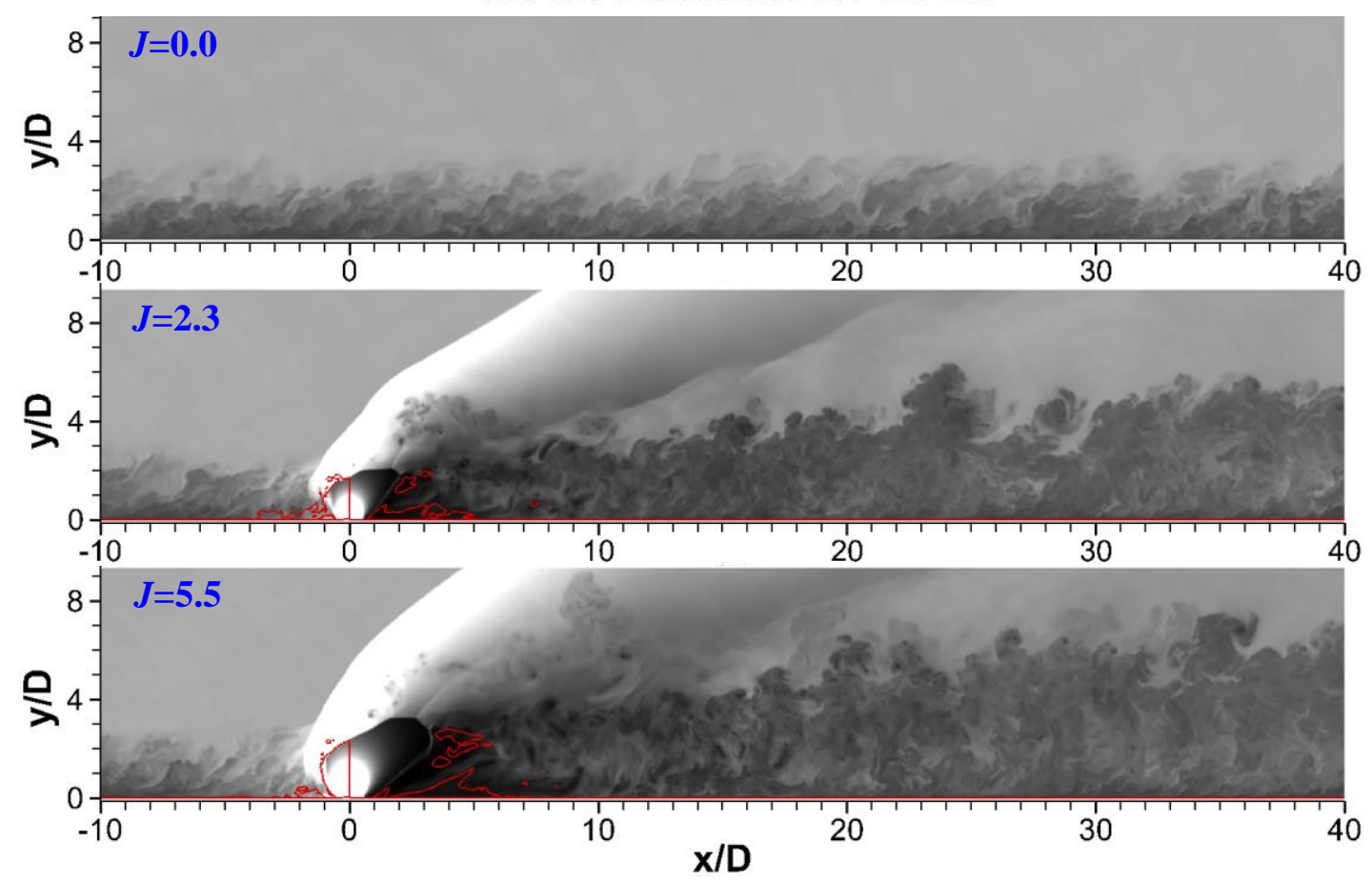

(a) Density contours at $z / D=0.0$ for $J=0,2.3,5.5$ (top to bottom). 

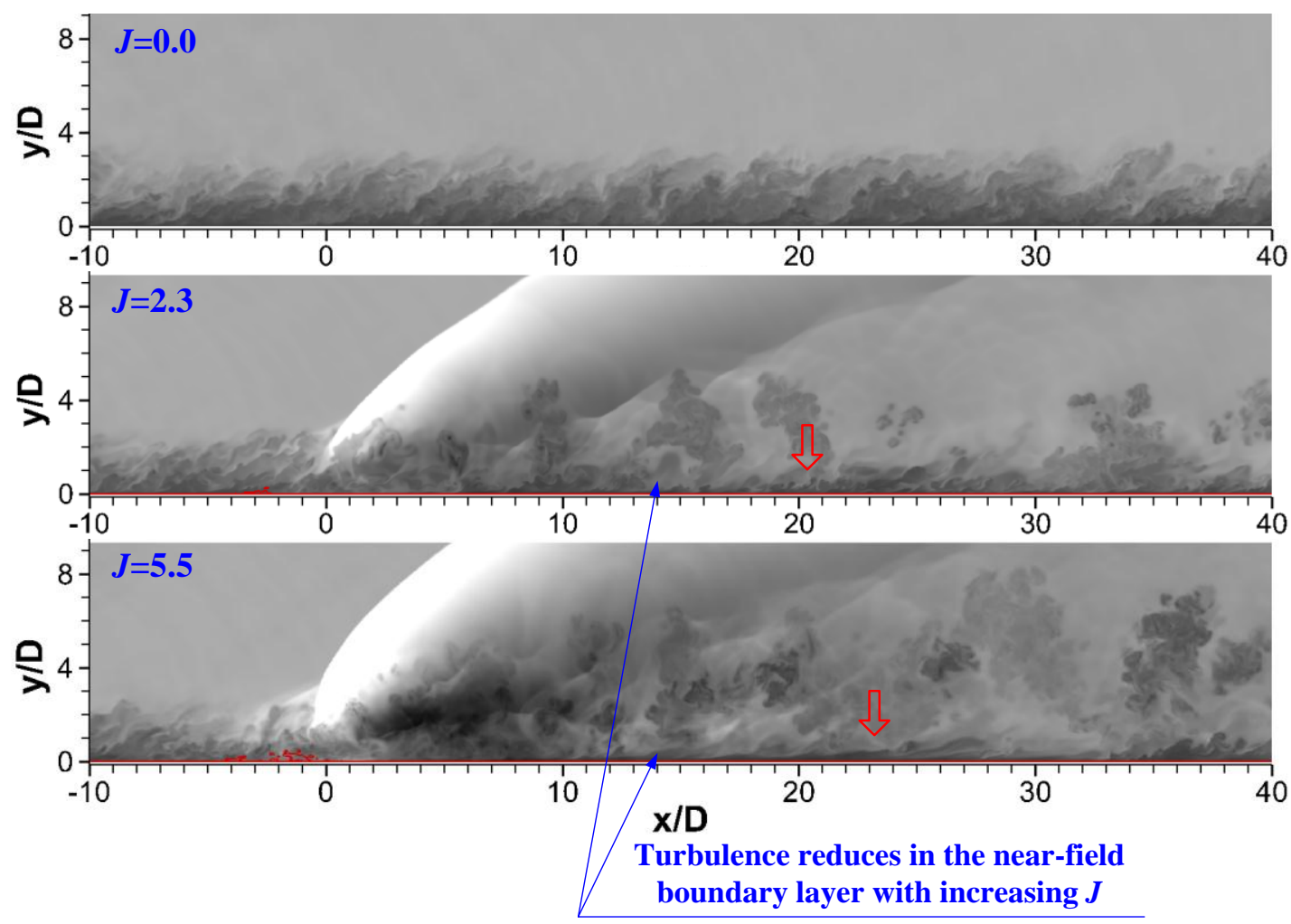

(b) Density contours at $z / D=3.0$ for $J=0,2.3,5.5$ (top to bottom).

FIGURE 2. Density contours at $z / D=0.0$ (a) and $z / D=3.0$ (b) planes of the jet interacting with the incoming boundary layer. The red solid lines are the isolines of $u / U_{\infty}=0.0$.
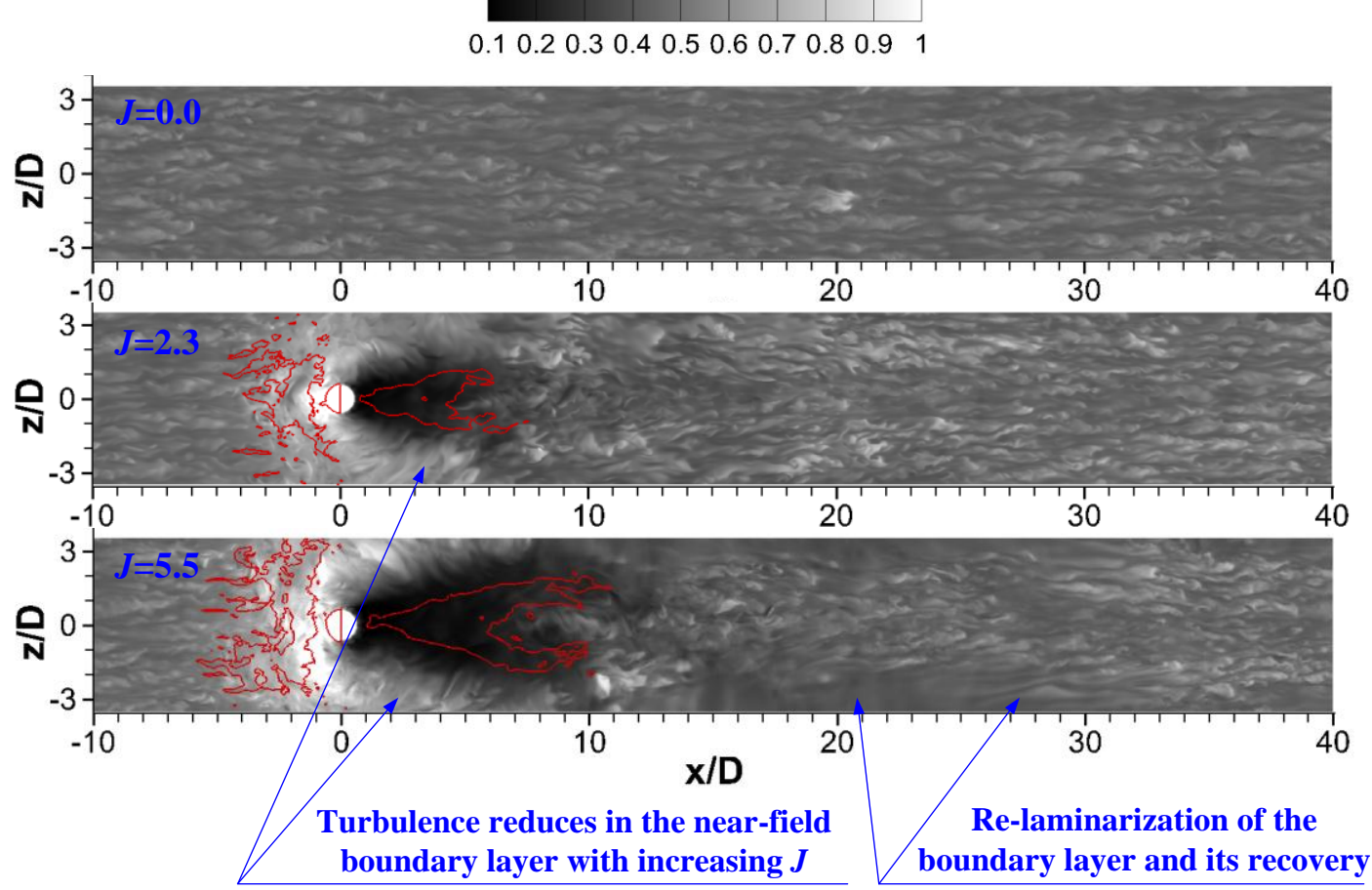

(a) Density contours at $y / D=0.08$ for $J=0,2.3,5.5$ (top to bottom). 

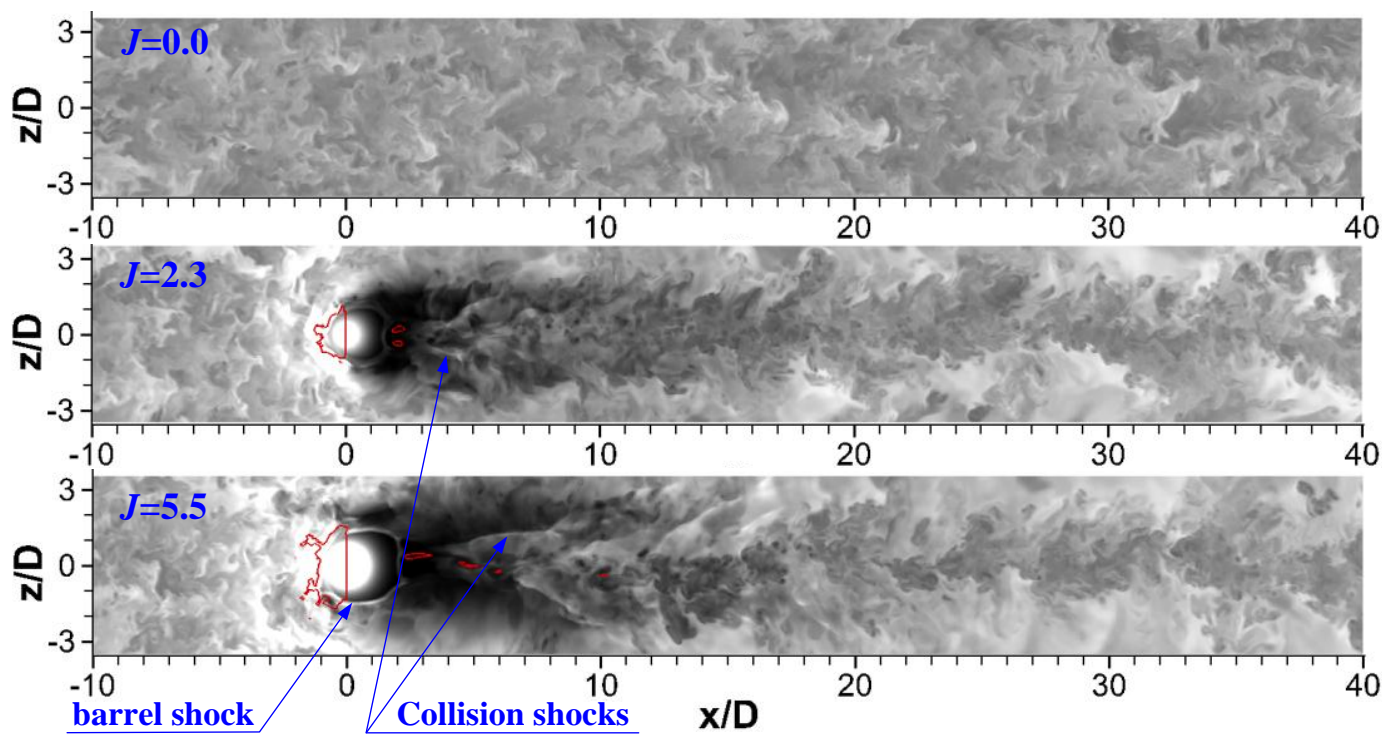

(b) Density contours at $y / D=1.0$ for $J=0,2.3,5.5$ (top to bottom).

FIGRUE 3. Density contours on $y / D=0.08$ (a) and $y / D=1.0$ (b) planes for different cases $(J=0,2.3$, 5.5 from top to bottom). The red solid lines are the iso-lines of $u / U_{\infty}=0.0$.

To clearly identify the development of the turbulence in the boundary layer downstream of the jet, the near-wall streaks in the turbulent flow field are shown and analyzed. The density contours at the same wall-normal distance $(y / D=0.08$ and $y / D=1.0)$ for $J=0,2.3$ and 5.5 are shown in figure 3 from a top view. Alternative low magnitude (dark color) and high magnitude (light color) density streaks can be identified in figure 3(a). The flow field of the jet interaction cases demonstrates different flow characteristics compared to the flat plate. We can see that the classic streaks are developed along the streamwise direction in the undisturbed boundary layer of the flat plate upstream of the jet orifices. For $J=2.3$ and $J=5.5$, the quasi-streamwise streaky structures crush into the separation region upstream of the jet. On the lateral of the jet, the streaks are interrupted by the bow shock induced by the jet. For both $J=2.3$ and $J=5.5$ cases, downstream of the jet lateral separation region, the coherent structures are greatly weakened and almost disappear, accompanied by increased density (shown in grey-white color). In the far-field of $x / D>10.0$, it can be seen from figure 3(a) that the boundary layer turbulence in the lateral downstream of the jet at 
$y / D=0.08$ of $J=2.3$ has a quick recovery and small streaks intermittently appear in the wall-bounded flow. For $J=5.5$, it is observed at $y / D=0.08$ that the turbulence decreases more obviously than $J=2.3$.

Figure $3(b)$ shows density contours on $y / D=1.0$ plane for all cases from a top view. Downstream of the jet lee, the high-speed flow impacts the shock waves which are generated by the collision of the flow running around the jet barrel. The collision shock waves are very clear in density contours on $y / D=1.0$ slices and they are twisted due to local turbulence. The boundary layer interacts with the shock generated by the collision of the flow around the jet barrel. It is clear that these collision shocks induce the herringbone separation shown in figure $3(a)$. It is seen that the herringbone separation region is confined and the height of the separation is limited since it decreases significantly in figure $3(b)$ at $y / D=1.0$. Flow over the separation reattaches downstream of the cross point of the separation branches. From figure $3(b)$, it is seen that the collision shock only exists in a limited region and the high speed flow in the lateral $(z / D>3.0$ or $z / D<-3.0)$ of the jet seems not to be affected by the shock waves. The flow behind the collision shock falls into the reattachment zone and after a certain distance the streaky structures re-organize near the symmetric plane, as shown in figure $3(a)$.

A comparison between figure $3(a)$ and $(b)$, especially for $J=2.3$, exhibits that at $y / D=0.08$ the streaks are regenerated early than $y / D=1.0$, which suggests that the turbulence in the inner layer of the lateral boundary layer recovers quickly than the outer layer. A comparison between $J=5.5$ and $J=2.3$ shows that higher $J$ leads to the intensified suppression on the wall-bounded flow. For $J=5.5$ the intensified suppression causes the lateral flow to stay in a weakened turbulence status that has not been fully recovered even near to the exit. For both $J=2.3$ and $J=5.5$ cases, the central region 
downstream of the effected region of the collision shock seems to remain in a turbulence status with high intensity since the streaks are abundant and corrugated. This phenomenon demonstrates that the collision flow in the jet lee leads to an amplification of the local turbulence in the boundary layer. A preliminary experimental observation on the slices of the flow field in Appendix B exhibits that figure 3 reflects the real flow property and reveals the validation of the simulation.

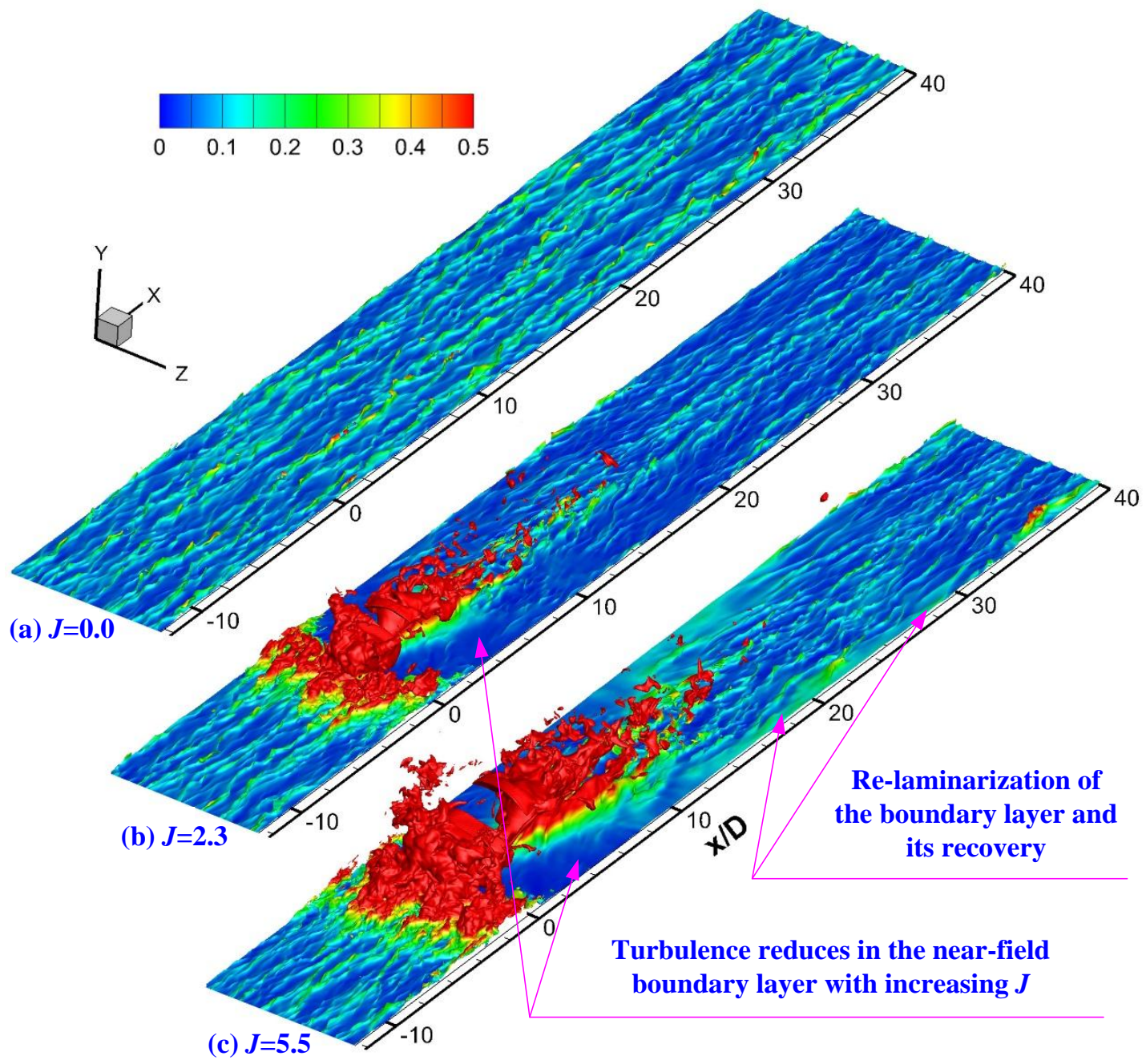

FIGURE 4. Iso-surface of the streamwise velocity $\left(u / U_{\infty}=0.4\right)$ fields of the jet interaction with the incoming boundary layer, (a) $J=0.0$, (b) $J=2.3$, (c) $J=5.5$. 


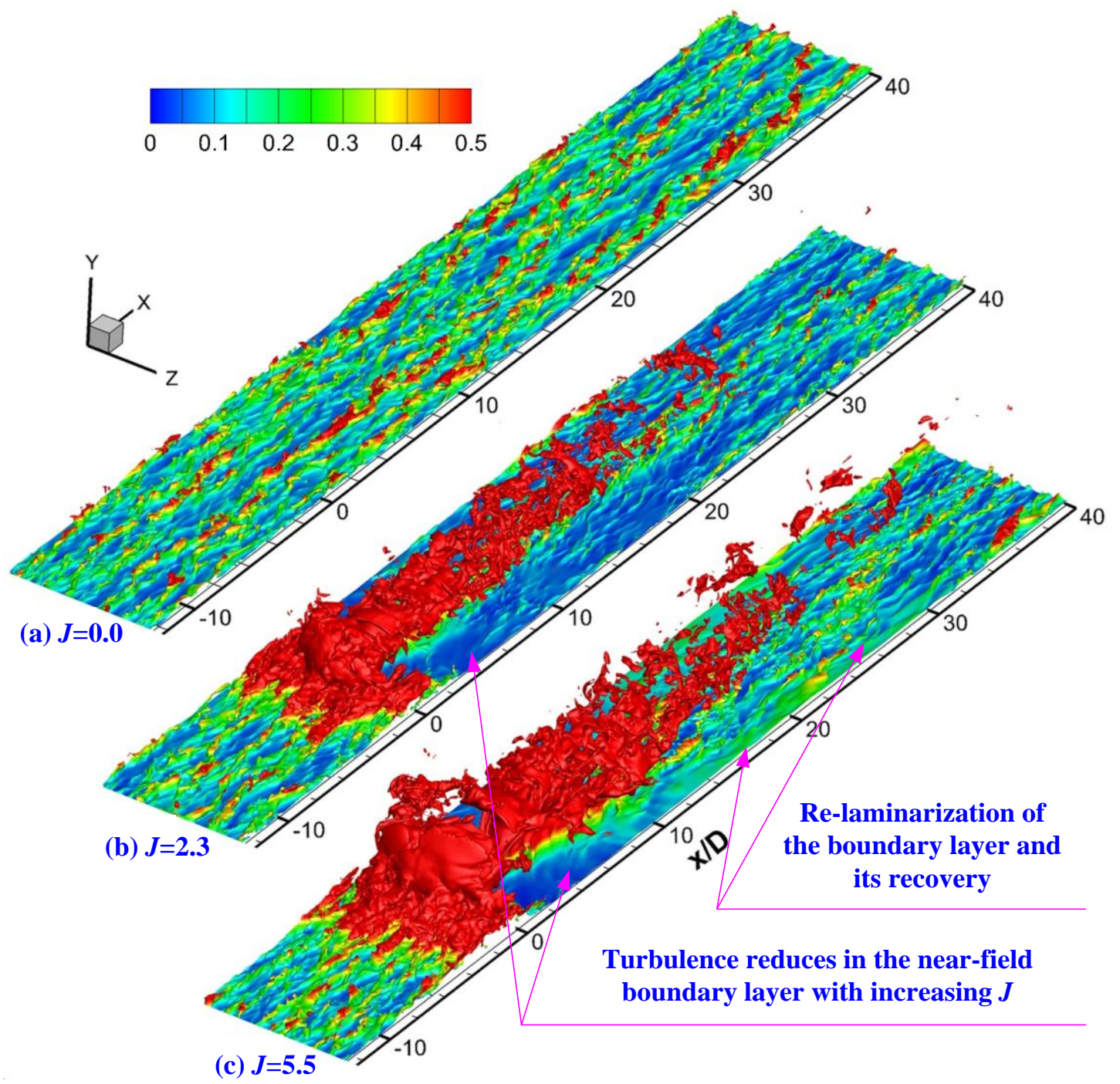

FIGURE 5. Iso-surface of the streamwise velocity $\left(u / U_{\infty}=0.55\right)$ fields of the jet interaction with

$$
\text { the incoming boundary layer, (a) } J=0.0 \text {, (b) } J=2.3 \text {, (c) } J=5.5 \text {. }
$$

Iso-surfaces of the streamwise velocity in the three dimensional flow field colored by the wall-normal distance are illustrated and compared in figures 4-5. For the flat plate $(J=0.0)$, we can clearly see the classic elongated streamwise vortices in the iso-surface of $u / U_{\infty}=0.4$, which keeps similar characteristics within the whole domain. A mean velocity of $u / U_{\infty}=0.4$ usually corresponds to an inner layer location in the boundary layer, as was shown in Sun, Hu \& Sandham (2017). The streaky structures concentrate in the inner part of the flat plate boundary layer and have no large liftup. For the $J=2.3$ and $J=5.5$ cases, the structures are similar to the flat plate case upstream of 
the jet. Further downstream the near-wall streaks develop into a completely new pattern which exhibits a suppression of the streaky structures. The phenomenon is especially prominent for the $J=5.5$ case. The iso-surfaces of $u / U_{\infty}=0.55$ given in figure 5 show that the large scale structures in the outer part of the boundary layer. Structures of the flat plate case (figure 5(a)) show that many packets grow from the near-wall region to a higher wall-normal location. These structures occur with the local mass and energy exchange between the outer layer and the main stream. In figure 5 (c) for the $J=5.5$ case, we can hardly see coherent structures in the lateral downstream of the separation bubble ahead of the jet orifice, which reflects a suppression of turbulence when flow goes across the jet windward separation region. Compared with the flat plate case, jet interaction modifies the boundary layer and weakens the exchange process of the low energy streaks in the inner layer with the outer layer. Downstream of the jet leeward separation at $x / D>10.0$, coherent structures in the near-wall region are regenerated during the recovery process and lifting large-scale structures in the outer part of the boundary layer keep growing along with the recovery. From the results shown in figure 4 and 5, we can find that the turbulence in the far-field is affected by the history effect of the turbulence decay in the vicinity of the jet. Higher $J$ leads to a more intensive interaction and a more apparent turbulence decay, which needs a longer distance to recover. In the jet leeward region near the centerline, turbulence is not suppressed since the jet interaction with the main stream enhances the local turbulence intensity, especially when the collision shock has an intensified interaction with the boundary layer.

\subsection{Mean flow properties and quantitative analysis on TKE and Reynolds shear stress}

This section further analyzes the turbulence formation mechanism in the boundary layer around the jet with turbulent statistics. Since averaging over 100 characteristic time is still not 
enough to obtain smooth profiles of high-order statistics, data are further averaged over the region $z \in[3.0 D, 3.5 D]$. It is assumed that the boundary layer is approximately homogenous in this spanwise direction and is not affected by the leeward collision shock waves, therefore averaging in the spanwise direction away from the jet is considered to be reasonable.

Profiles normal to the wall are extracted at several locations upstream and downstream of the jet to compare with the results at the same locations of the flat plate case, as is illustrated in figure 6 for the streamwise velocity. The region in the vinicity downstream of the jet corresponds to the strong interaction area underneath the jet with the boundary layer. It can be seen that for the jet cases, the velocity downstream of the jet is larger over most of the boundary layer than the flat plate case, and the downstream velocity profiles become fuller as $J$ increases. It is interesting that at $x / D=5$ the inner layer velocity $(y / D<0.15$, shown in figure $7(a))$ of $J=5.5$ is larger than $J=2.3$ and $J=0.0$, while at $x / D=35$ the inner layer velocity $(y / D<0.15$ shown in figure $7(b))$ of $J=0.0$ is basically same with $J=5.5$ and $J=2.3$. This implies that in the far-field downstream of the jet, the inner-layer velocity for $y / D<0.15$ of $J=2.3$ and $J=5.5$ almost recovers to the shape of the fully-developed turbulent boundary layer of $J=0.0$ case (flat plate), while the outer layer still stays in the status that the profile is not fully recovered.

Figure 8 gives the boundary layer 99\% thickness $\delta_{99}$ predictions along the streamwise direction for all cases. $U_{\mathrm{e}}$ is taken as the free-stream velocity at $y / D=3.0$. This is not an accurate estimation but it provides a useful comparison. It is not surprising to see the boundary layer thickness of the jet cases has an obvious drop downstream of the jet. Since there is a strong interaction region at $4.0<x / D<20.0$, an inaccurate prediction for the boundary layer thickness is shown in figure 8. After $x / D=20.0$, it is seen the boundary layer thickness of the jet cases is 
smaller than the flat plate case, which reflects the decrease of the boundary layer thickness in the jet lateral downstream of the windward separation. The boundary layer thickness even drops below $0.8 D$ after the jet for the $J=5.5$ case. This result corresponds to the experimental NPLS images given in Appendix B where the boundary layer thickness decreases in the jet lateral.

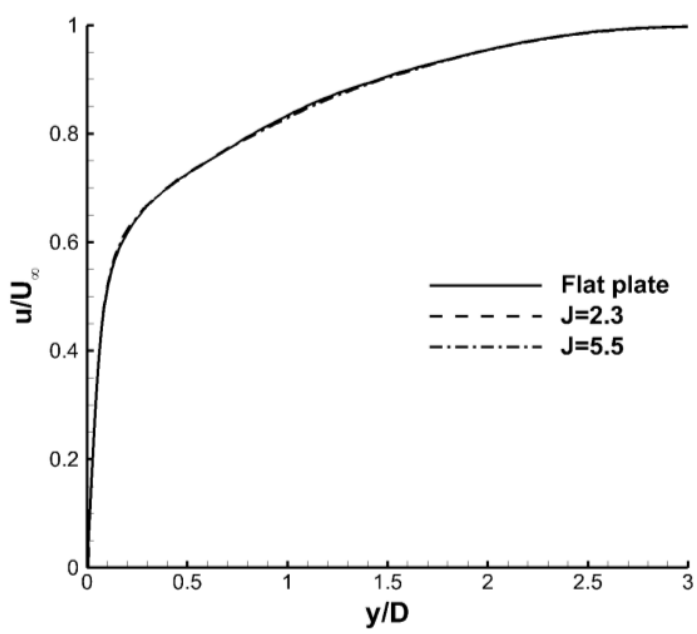

(a) $x / D=-10.0$

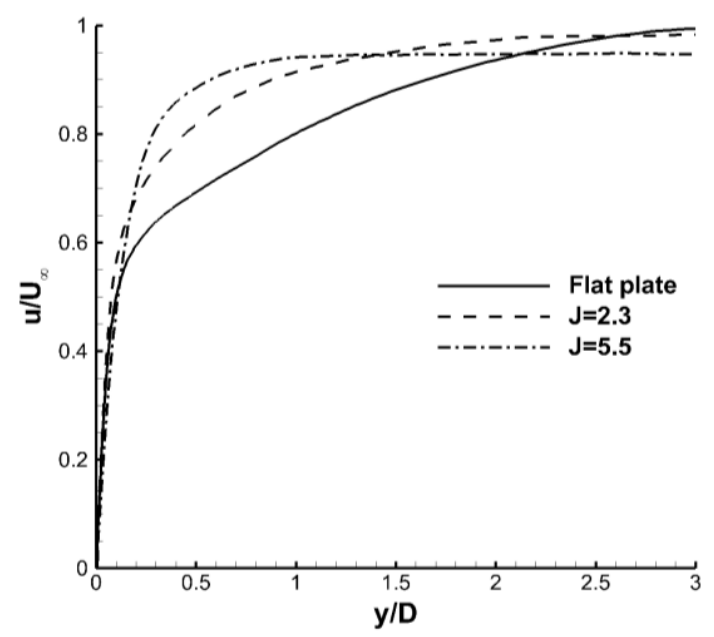

(c) $x / D=20.0$

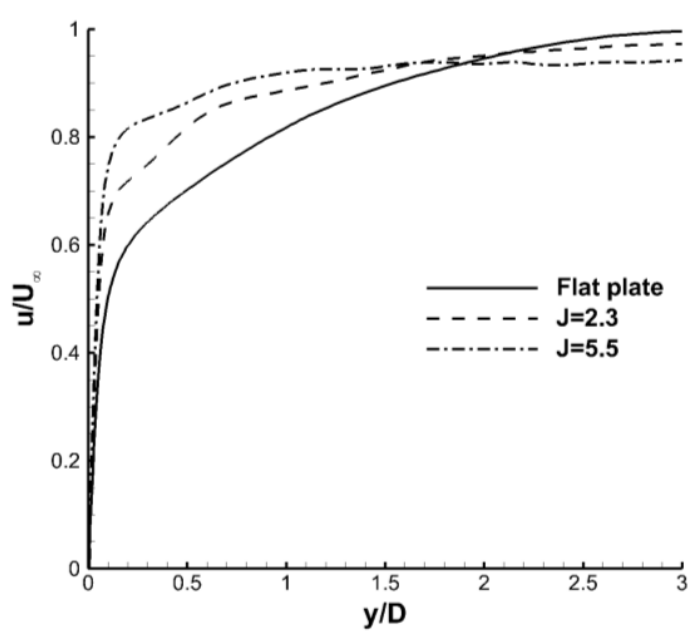

(b) $x / D=5.0$

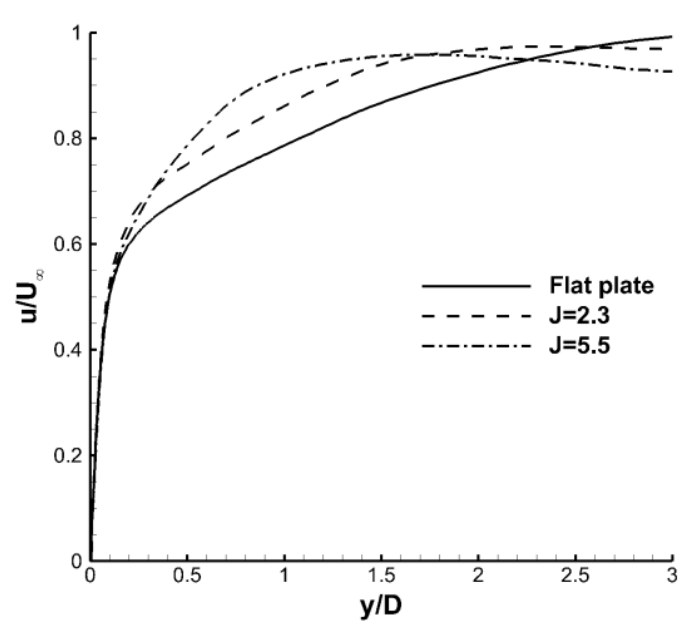

(d) $x / D=35.0$

Figure 6. Mean velocity profiles at different streamwise locations, (a) $x / D=-10.0$, (b) $x / D=5.0$, (c) $x / D=20.0$, (d) $x / D=35.0$, normalized by inflow free-stream velocity. 


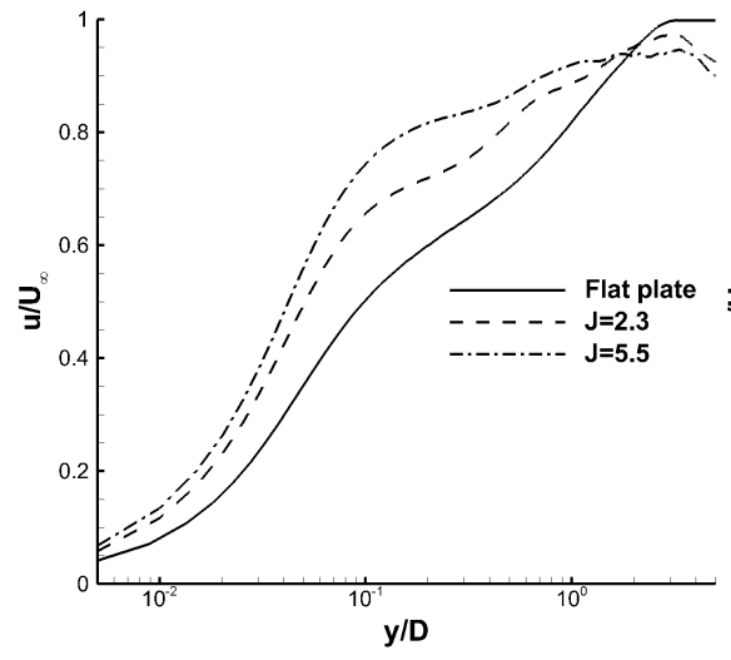

(a) $x / D=5.0$

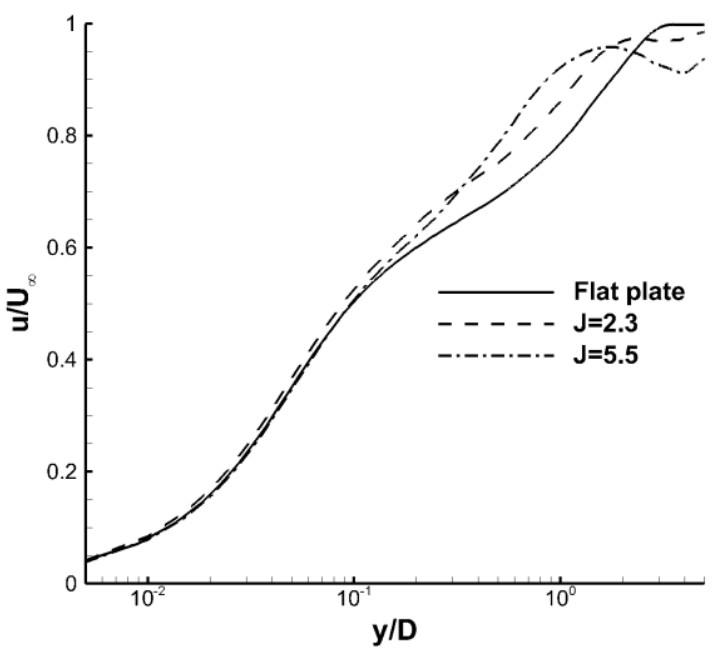

(b) $x / D=35.0$

FIGURE 7. Mean velocity profiles at different streamwise locations in the logarithmic coordinate,

normalized by the inflow free-stream velocity.

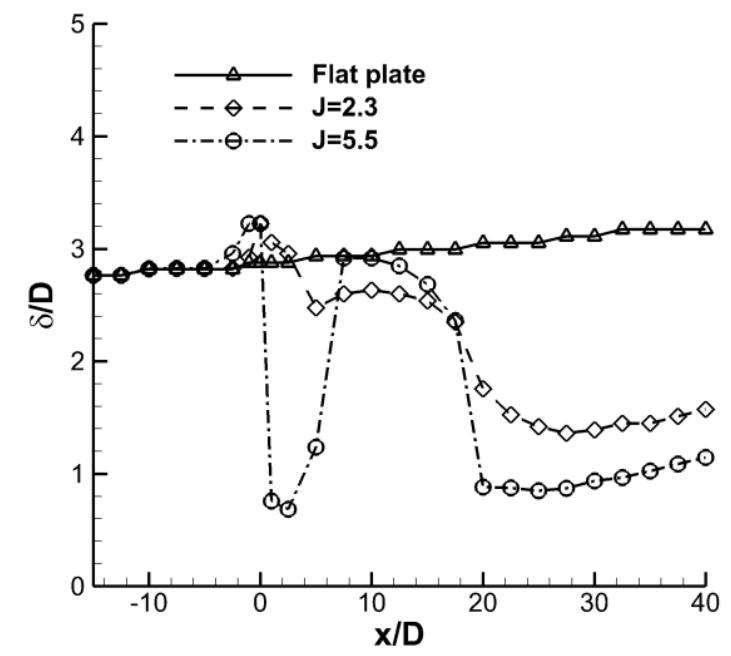

FIGURE 8. Boundary layer thickness $\delta_{99}$ predictions along the streamwise direction, $U_{\mathrm{e}}$ is taken as the free stream velocity at $y / D=3.0$

Time-averaged skin friction along the wall in the domain $z \in[3.0 D, 3.5 D]$ is shown in figure 9 .

For an incompressible flow, if assuming the mean velocity profile follow $u / U_{e}=(y / \delta)^{1 / 7}$, the skin friction considering compressibility effects can be calculated, following Young's assumption (Young 1989), as $c_{f} / c_{f i} \approx\left(1+0.1165 M_{\infty}^{2}\right)^{-0.622}$ for adiabatic wall, where $c_{f i}$ denotes the skin friction 
at the same Reynolds number in the incompressible flow. If we calculate $R_{\text {ex }}$ from $\delta^{v d}=0.37 x \operatorname{Re}_{x}^{-0.2}$ and $c_{f i}$ is obtained as $c_{f i}=0.0592 \operatorname{Re}_{x}^{-0.2}$, we get $c_{f} \approx 0.00209$ at $x / D=-7.5$ for the flat plate. The prediction is comparable to the numerical calculation $c_{f} \approx 0.00188$. The skin friction for different cases follows the theoretical result of a flat plate turbulent boundary layer before $x / D=-10.0$, which indicates that the artificial inflow condition develops to a fully turbulent boundary layer before the jet. The skin friction coefficient decreases suddenly over $-10.0<x / D<-2.0$ in the separation region ahead of the jet. In the region $-2.0<x / D<0$, the skin friction coefficient recovers across the jet lateral separation. The wall pressure (shown in figure $9(b))$ rises in this region is due to the bow shock effect. Downstream of the location $x / D=-1.0$ for $J=2.3$ and $x / D=-2.0$ for $J=5.5$, wall pressure falls corresponding to an expansion in the supersonic flow. It is worth mentioning that the wall pressure starts to decease ahead of the jet, which means the expansion starts upstream of the jet, not only in the jet lee, as seen in figure $9(b)$. For $x / D>7.5$, the wall pressure gradient is positive, which corresponds to the recovery process. In the region $3.0<x / D<8.0$ for $J=2.3$ and $3.0<x / D<10.0$ for $J=5.5$, the skin friction coefficient decreases and reaches a local minimum. Downstream of this region, the skin friction quickly recovers towards an equilibrium status for $J=2.3$, while a slower recovery exists for $J=5.5$. At the location $x / D=40.0$ near the physical domain exit, the skin friction coefficient for both jet cases approaches the flat plate value, which indicates that the inner layer flow almost recovers to the fully-developed turbulent boundary layer. These results are consistent with the analysis for streaks shown in figures 3-5 and velocity profiles in figures 6-7. It is identified that the expansion process exists across the jet separation bubble. As is well known, a sudden expansion suppresses the turbulence in the supersonic flows (Sun, Hu \& Sandham 2017), which could be the reason that the streaks are 
weakened and the skin friction coefficient falls.

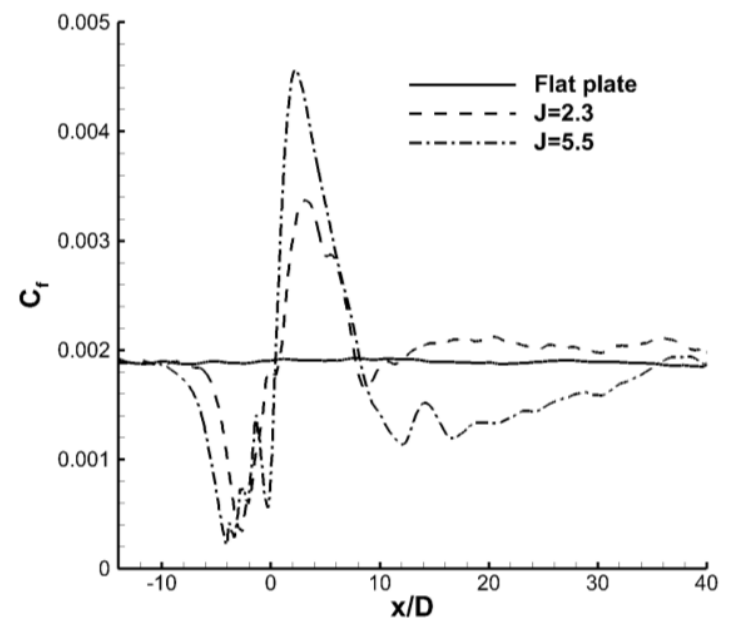

(a) Skin friction coefficient

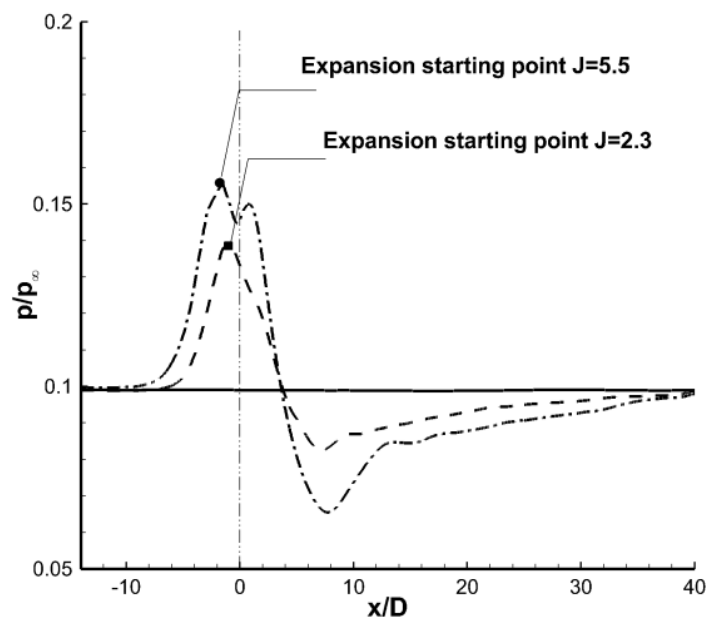

(b) Wall pressure distributions

FIGURE 9. Skin friction coefficient (a) and wall pressure (b) distributions along the wall averaged for the zone $z \in[3.0 D, 3.5 D]$.

Further analysis of the mean flow is presented in figures 10-11, showing contours of static pressure and Mach number at various locations. It is seen that the pressure downstream of the separation region in front of the jet decreases until the flow runs to the collision shock waves. Streamlines on the Mach number contours shown in figure 10 reflect a divergence in the lateral around the jet which corresponds to an expansion. A low pressure zone exists in the jet lateral due to the blockage and expansion effects from the jet. The flow in the jet lateral is supersonic and the collision shock is induced. Cross-flow runs around the jet and impinges together in the jet lee, which leads to a local pressure increment due to the collision effects. Recall the turbulence reduction phenomenon in a supersonic boundary layer flow over an expansion corner (Arnette, Samimy \& Elliott 1998), it is indicated that the sudden expansion would lead to the local turbulence decay. The separation region after the collision shocks in the jet lee on $y / D=0.08$ plane is large, however almost disappears on $y / D=0.5$, which means the height of the separation zone is smaller than $y / D=0.5$. The recirculating flow within the separation zone on the $y / D=0.08$ slice 
reattaches in a $\mathrm{V}$-shape reattachment region. The supersonic flow at $y / D=0.5$ slice around the jet impinges into a concentrated flow behind the collision shocks. The reattached supersonic flow and the impinging flow near the wall leads to a pressure increment in the downstream region of the leeward separation bubble, as shown in figure $10(a)-(b)$.

Turbulent kinetic energy (TKE) contours on different wall-parallel planes for $J=5.5$ are plotted in figure 11, which shows the whole flow field including the jet wake and the far-field. It is seen that TKE in the separation region ahead of the jet is increased compared to TKE upstream on both $y / D=0.08$ and $y / D=0.5$ planes. Downstream of the windward separation bubble, TKE reduces significantly, which corresponds to the process of the lateral supersonic flow running around the jet orifice. In the jet far-field, TKE remains at a lower level due to the history effects of the near-field turbulence decay. The herringbone separation zone behind the collision shocks has a lower TKE level due to the recirculating flow. Outside of the herringbone zone, TKE increases obviously due to the shock interaction with the boundary layer and the reattachment of the jet leeward flow. Above the herringbone zone $(y / D>0.5)$, TKE is intensified due to the interaction of the collision shocks and the flow impingement. In the region downstream in the jet far-field, TKE is higher in the centerline than that on the lateral along the span.
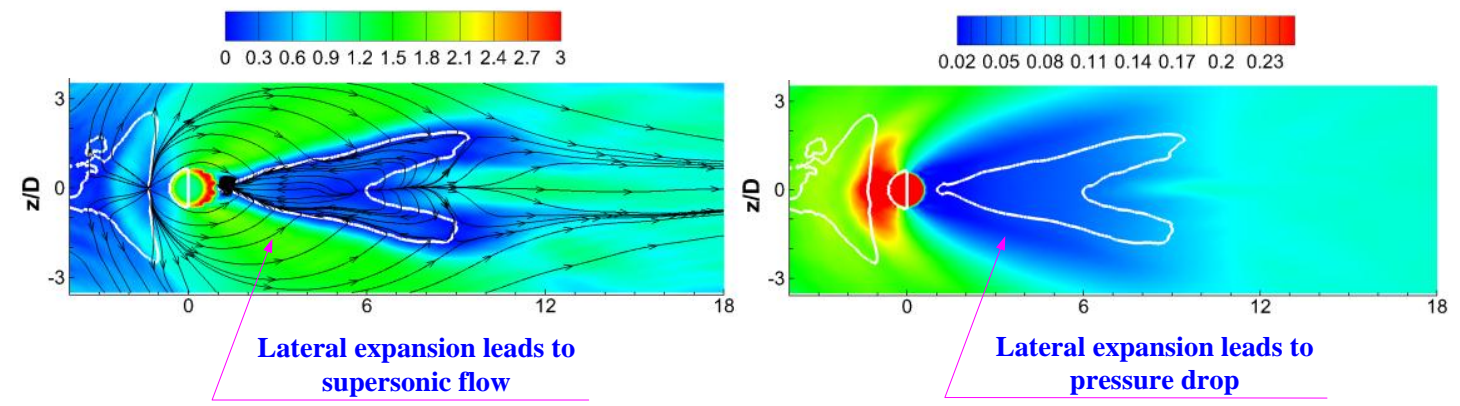

a) $y / D=0.08$ 

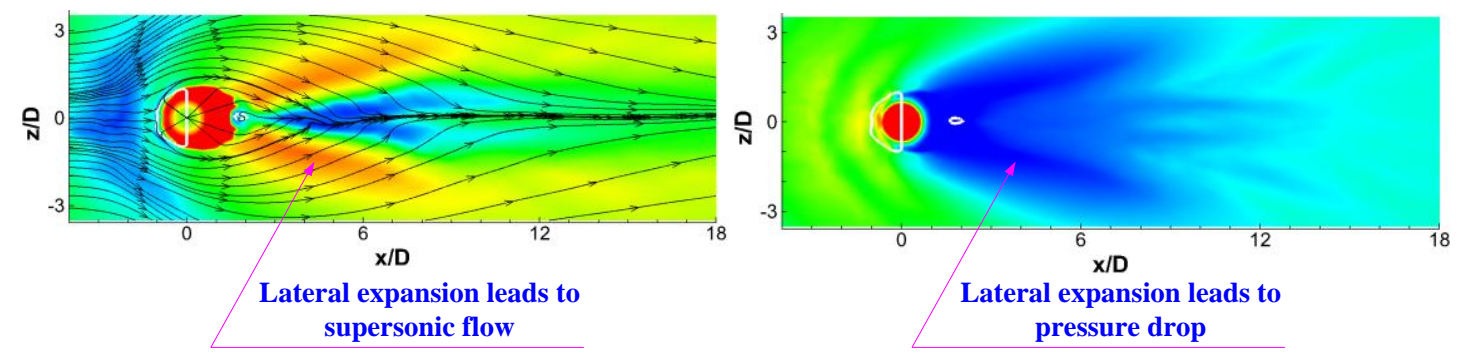

b) $y / D=0.5$

FIGURE 10. Contours of the Mach number (left) and static pressure (right) on different

wall-parallel planes for the $J=5.5$ case. Streamlines are superimposed on the Mach number

contours. The white solid line superimposed on the contour plots represents $u / U_{\infty}=0.0$.

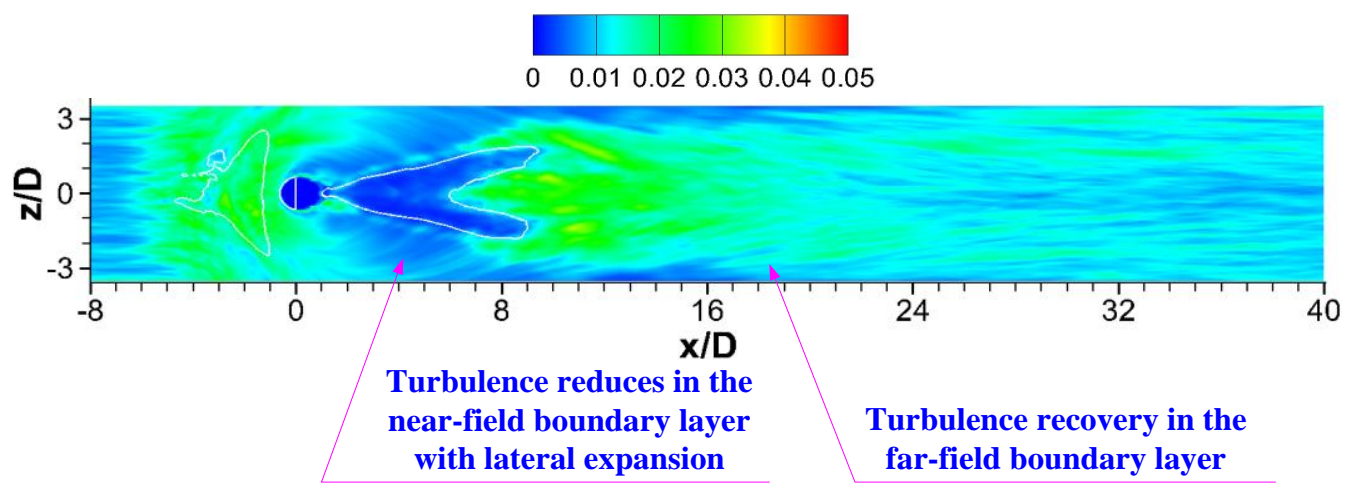

(a) $y / D=0.08$

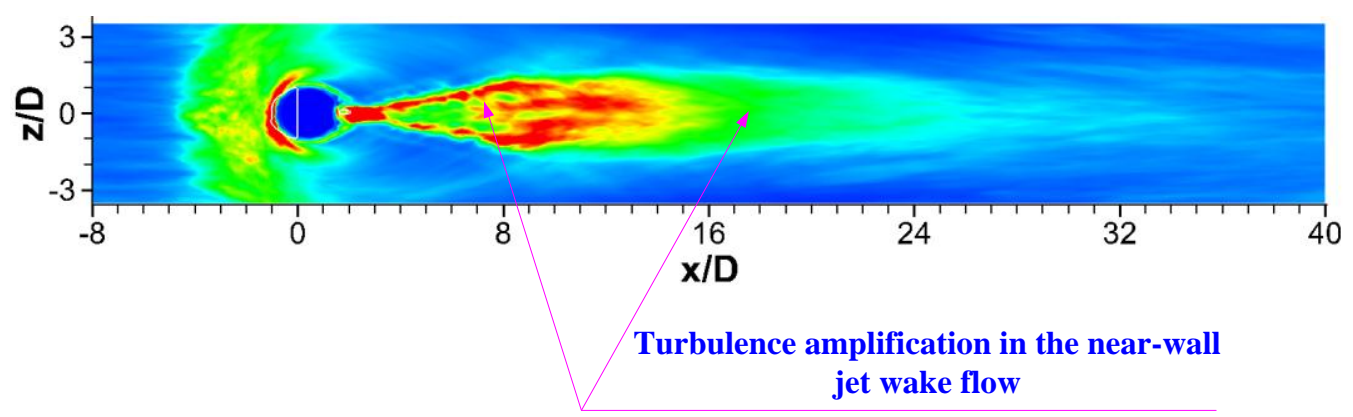

(b) $y / D=0.5$

FIGURE 11. TKE contours on different wall-parallel planes of the $J=5.5$ case. The white solid line superimposed on the contour plots represents $u / U_{\infty}=0.0$.

Representative TKE profiles averaged over $z \in[3.0 \mathrm{D}, 3.5 \mathrm{D}]$ along the streamwise direction are 
given in figures 12-13. Results of the flat plate case are also plotted for a comparison. At $x / D=5.0$, TKE is much higher for both jet cases due to the intensive interaction of the jet with the boundary layer flow. As is shown in figure 11 , TKE at $x / D=5.0$ is smaller than that within the zone of $-2.0<x / D<3.0$ since the flow over the windward separation region has a higher TKE, which indicates TKE reduction occurs after the windward separation. It is also seen that the jet lateral supersonic streamlines diverge in figure 10 at $x / D=5.0$, which corresponds to the expansion process and leads to the turbulence decay. At $x / D=20.0$, TKE reduction continues and a ravine with low TKE magnitude forms between the inner layer and the outer layer. This phenomenon is more obvious for the $J=5.5$ case (see where the blue arrow points in figure $12(d)$ ) at $x / D=35.0$ although the boundary layer is recovering. In the inner layer, TKE is increased for both jet cases compared to the flat plate case, which indicates that the near wall turbulence remains at a higher level after the flow goes across the jet windward separation region. The ravine in the TKE profile is very clear compared to the flat plate case, which reveals a significant TKE decay near the inner layer. In the outer layer, the interaction between the jet and the boundary layer flow exists and the local TKE magnitude deviates from the flat plate case significantly. As the boundary layer grows, the TKE ravine extends to the outer layer due to the history effect of the turbulence suppression near the jet orifice. Recall the analysis for figures 2-5, the streaky structure disappearance reflects the local turbulence laminarization, which originates from the significant turbulence decay near the jet orifice. TKE in the boundary layer downstream of the jet averaged over the region $z \in[-0.5 D, 0.5 D]$, shown in figure 13 , is kept at a high level due to the interaction of the collision shock and the impingement of the lateral supersonic flow running around the jet, as analyzed for figures 10-11. Corresponding to figure 2-5, along the streamwise domain within $z \in[-0.5 D, 0.5 D]$, 
abundance of turbulence is generated and the turbulent vortices create the high level TKE downstream of the jet.

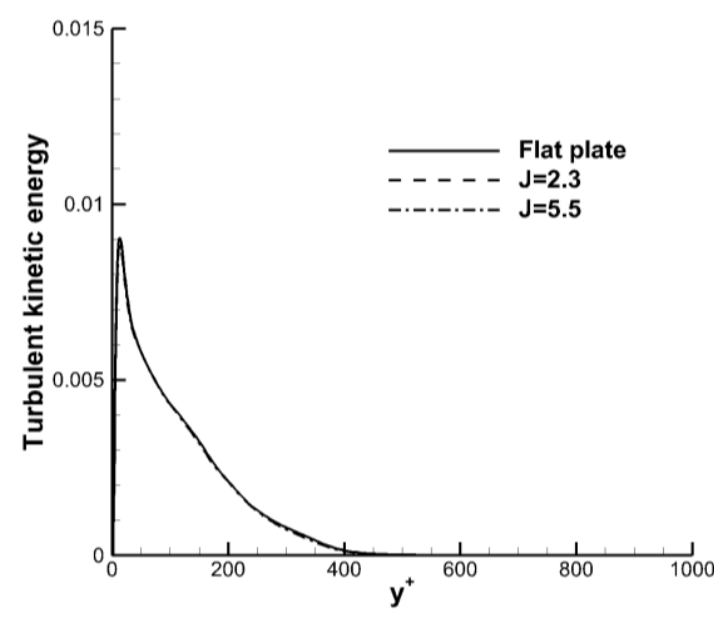

(a) $x / D=-10.0$

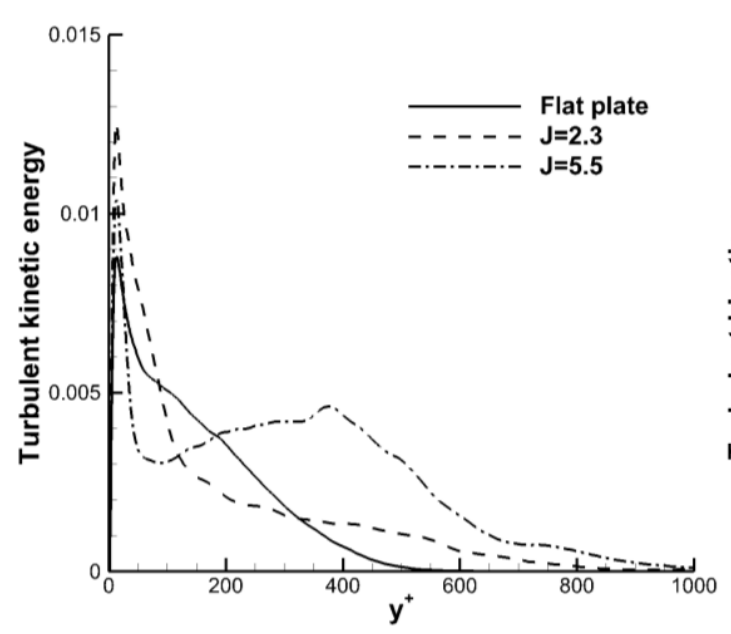

(c) $x / D=20.0$

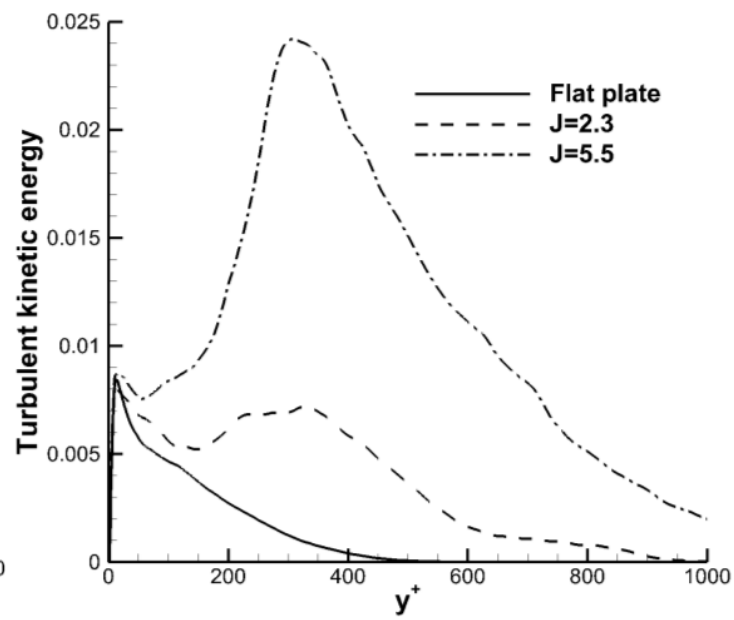

(b) $x / D=5.0$

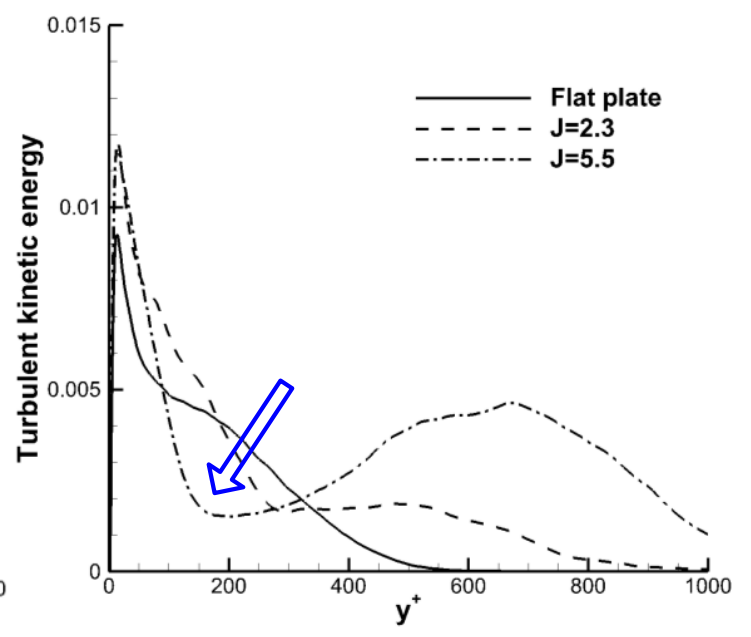

(d) $x / D=35.0$

FIGURE 12. TKE profile predictions at different locations along the belt $z \in[3.0 D, 3.5 D]$, (a) $x / D=-10.0$, (b) $x / D=5.0$, (c) $x / D=20.0$, (d) $x / D=35.0$, normalized by the square of the inflow free-stream velocity. 


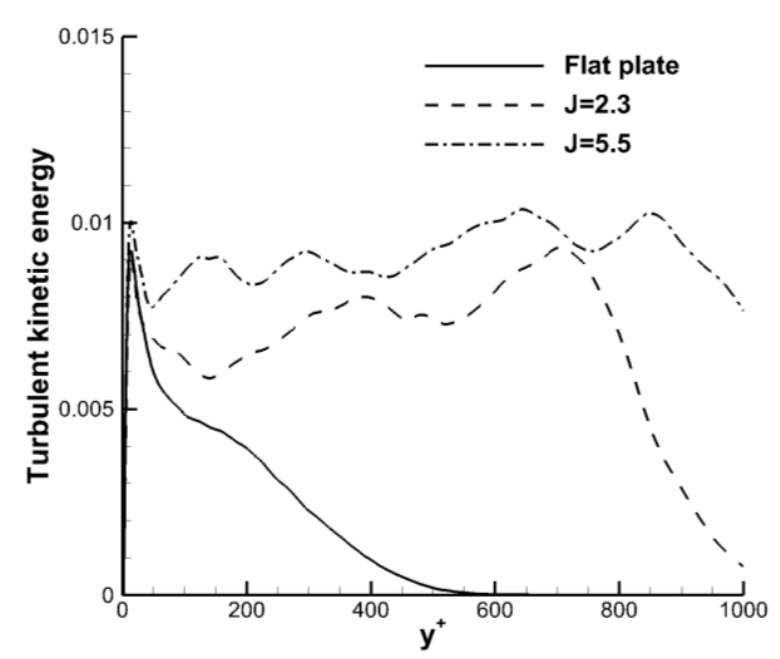

FIGURE 13. TKE profiles at $x / D=35.0$ averaged over $z \in[-0.5 D, 0.5 D]$, normalized by the square of the inflow free-stream velocity.

Figure 14 shows the evolution of RMS velocity components along the streamwise direction for the $J=5.5$ case compared with the profiles on the flat plate. At $x / D=5.0$, all velocity fluctuations are suppressed in the inner layer of $y^{+}\left\langle 20\right.$ except $\langle w\rangle_{\mathrm{RMS}}$ which is increased. In the outer layer, turbulence is enhanced significantly due to the interaction of the jet with the boundary layer flow. Comparing the RMS velocity components at $x / D=20.0$ and $x / D=35.0$, it is clearly seen that the turbulence in the inner layer recovers to the same level with the flat plate flow; however, it is still not recovered thoroughly due to the history effect of the turbulence decay in the vicinity of the jet. It is obvious that a ravine (see where blue arrow points in figure $14(d)$ ) exists in the profile of the shear stress profile even at $x / D=35.0$ compared to the flat plate case. Since the shear stress is mainly associated with large-scale structures, it is consistent with the result that the large structures are less coherent downstream of the jet. The reduction of the turbulence level between the inner and the outer layer highlights a weakening of the large-scale structures in the jet lateral flow. Due to the turbulence suppression in this region, the exchange of turbulence energy between the inner layer and the outer layer is reduced, which results in a turbulent energy accumulation in 
the inner layer since the turbulence in the inner layer exists in a local equilibrium, as seen in the profiles of figure 12. The turbulence energy in the inner layer cannot be transferred to the outer layer due to the suppressed exchange. The RMS velocity components at $x / D=35.0$ averaged in $z \in[-0.5 D, 0.5 D]$ are kept at a high level and no significant deviation exists in the profiles of $J=5.5$ case compared to flat plate case, as shown in figure 15, which indicates the turbulence stress stays in a strong intensity along the streamwise domain within $z \in[-0.5 D, 0.5 D]$ downstream of the jet.

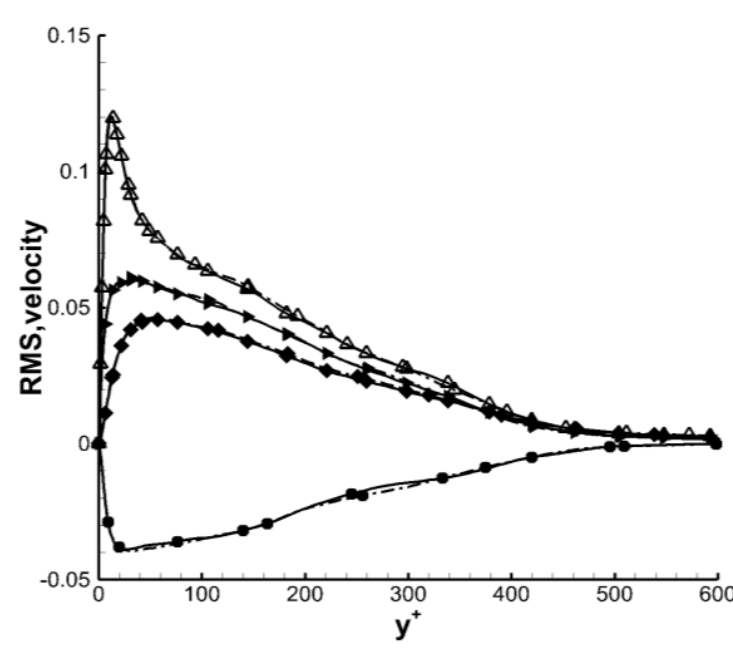

(a) $x / D=-10.0$

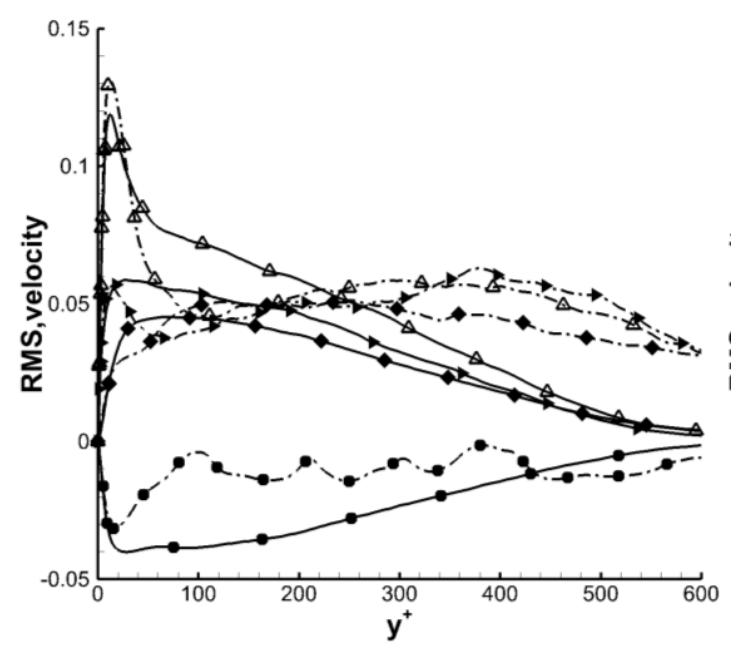

(c) $x / D=20.0$

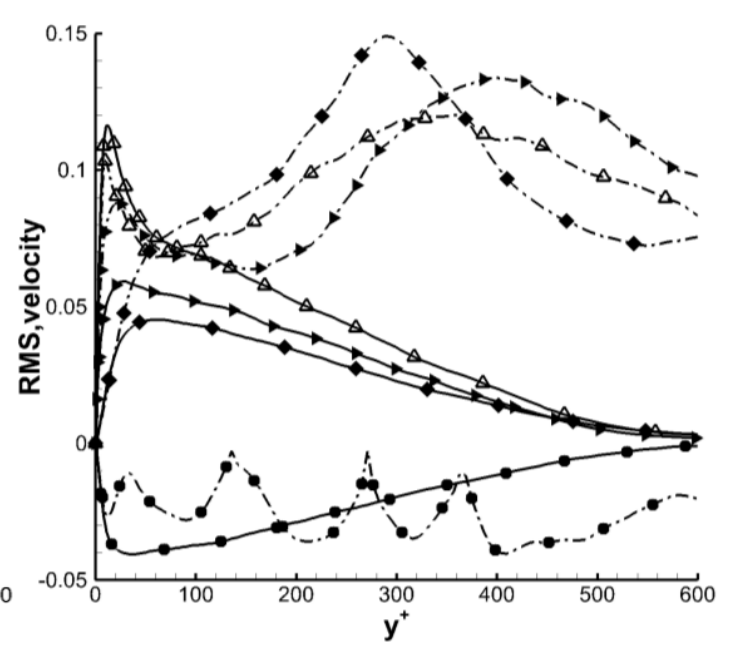

(b) $x / D=5.0$

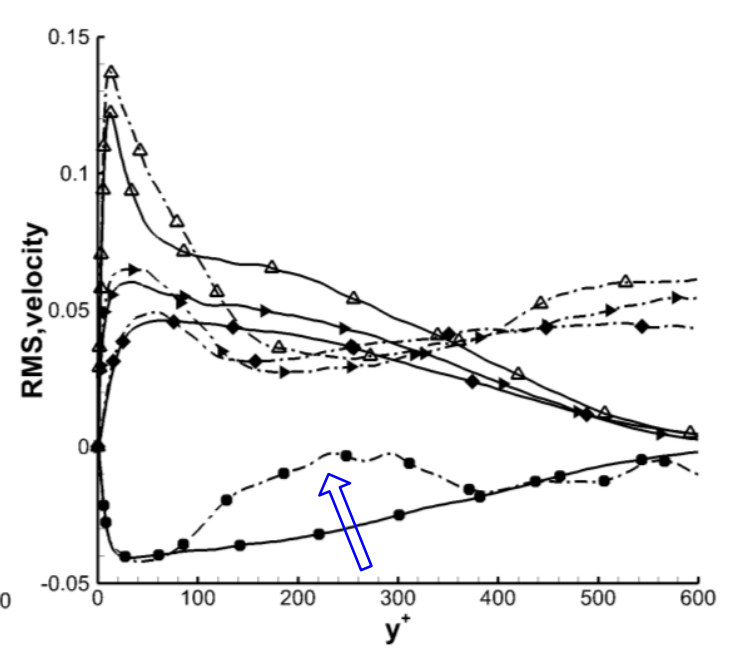

(d) $x / D=35.0$

FIGURE 14. RMS velocity profiles at different locations averaged over $z \in[3.0 \mathrm{D}, 3.5 \mathrm{D}]$, plotted in wall unit coordinate, (a) $x / D=-10.0$, (b) $x / D=5.0$, (c) $x / D=20.0$, (d) $x / D=35.0$, normalized by the inflow free-stream velocity. Solid lines represent the flat plate case, and dashdotted lines represent 
the $J=5.5$ case. RMS velocities of the streamwise $(\Delta)$, wall-normal $(\bullet)$ and spanwise components, together with the Reynolds shear stress $(\bullet)$.

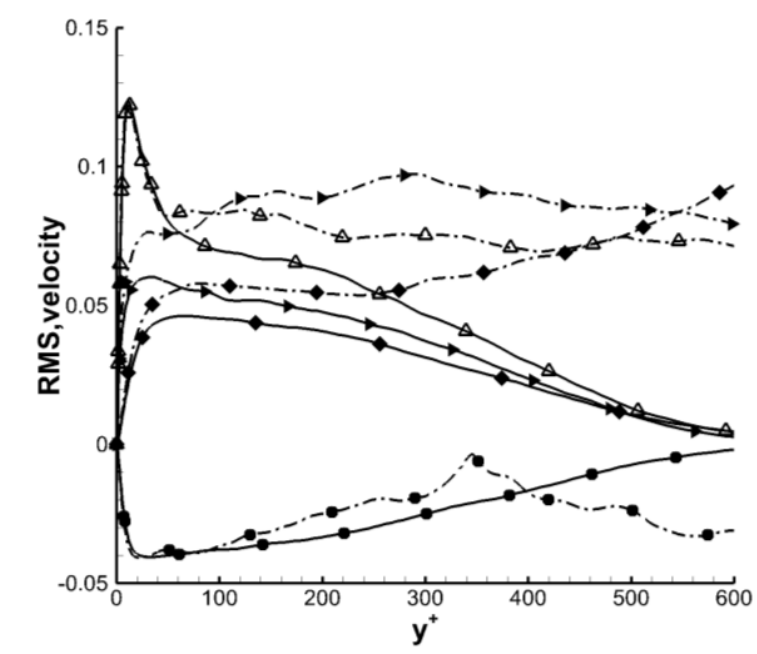

FIGURE 15. RMS velocity profile predictions at $x / D=35.0$ in the belt $z \in[-0.5 D, 0.5 D]$,

normalized by the inflow free-stream velocity. Solid lines represent the $J=5.5$ case and dashdotted lines represent the flat plate case for RMS velocities of the streamwise $(\Delta)$, wall-normal $(\diamond)$ and spanwise $(\longrightarrow)$ components, together with Reynolds shear stress $(\bullet)$.

TKE and the shear stress $\left\langle u^{\prime} v^{\prime}\right\rangle$ and their evolutions along $y / D=0.08$ and $y / D=0.6$ in the domain $z \in[3.0 D, 3.5 D]$ are shown in figure 16 and 17 , respectively, from which a similar trend of the different evolution processes for jet cases can be seen. The amplification of the peak values of all components is due to the interaction of jet with the boundary layer, which corresponds to the windward separation region in front of the jet. The TKE magnitudes start to decrease within the separation region. It is interesting to note that the absolute values of both TKE and $\left\langle u^{\prime} v^{\prime}\right\rangle$ start to decease ahead of the jet, which corresponds to the analysis of pressure distribution in figure $9(b)$. From figures 16-17, we can see that the evolution of TKE and $\left\langle u^{\prime} v^{\prime}\right\rangle$ depends on the momentum flux ratio $J$. As $J$ increases, the peak values of TKE and $\left\langle u^{\prime} v^{\prime}\right\rangle$ decrease in the lateral downstream of the jet. $\left\langle u^{\prime} v^{\prime}\right\rangle$ is reduced to almost zero along $y / D=0.6$ for $J=5.5$, indicating that turbulence is 
greatly suppressed and even laminarization happens. The magnitude of TKE and $\left\langle u^{\prime} v^{\prime}\right\rangle$ decreases to local minima soon after the separation at $y / D=0.08$, then gradually re-develops outwards. Along $y / D=0.08$ downstream of the jet orifice, the peak of TKE and $\left\langle u^{\prime} v^{\prime}\right\rangle$ first occurs around $x / D=18.0$ for $J=2.3$, where the inner layer is basically recovered. While at $y / D=0.6$ for $J=2.3$, the peak of TKE and $\left\langle u^{\prime} v^{\prime}\right\rangle$ first occurs around $x / D=25.0$, suggesting the outer boundary layer takes a longer distance to recover than the inner layer. Compared to $J=2.3$, the peak of TKE and $\left\langle u^{\prime} v^{\prime}\right\rangle$ of $J=5.5$ appears further downstream, which means that wall turbulence regeneration in the near-wall region is slower for higher $J$. For $J=2.3$, the process of the regeneration of wall turbulence is within a shorter distance than $J=5.5$, which can be again attributed to the interaction induced by the jet plume with the boundary layer.

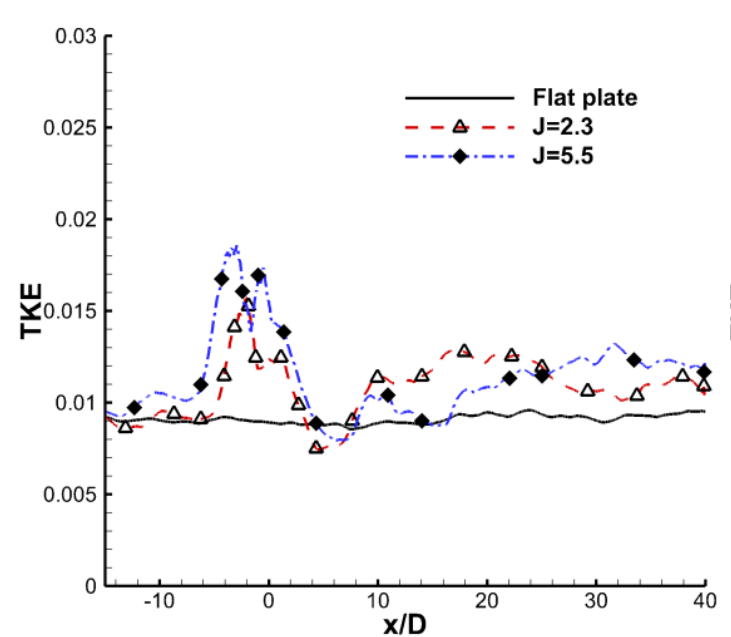

(a) $y / D=0.08$

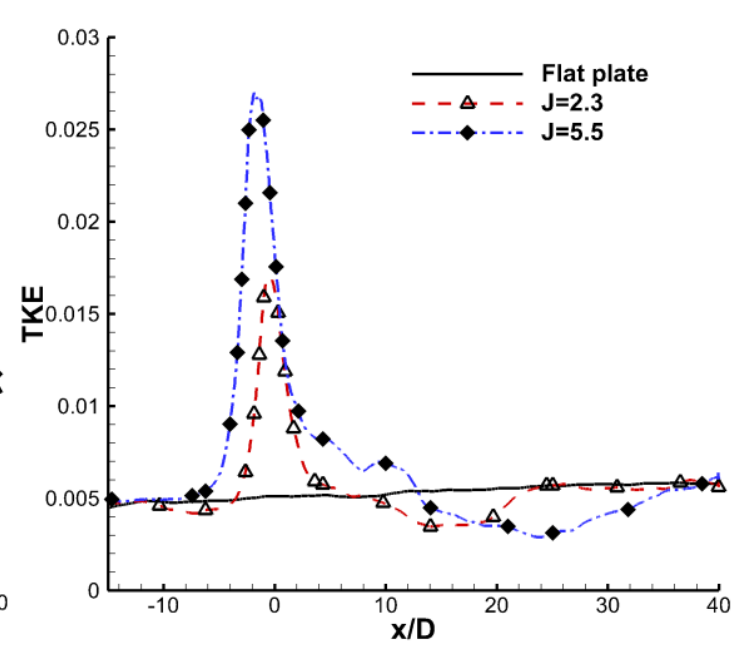

(b) $y / D=0.6$

FIGURE 16. TKE along streamwise direction at $y / D=0.08$ and $y / D=0.6$ in the domain $z \in[3.0 D$,

$3.5 D$ ] of different cases, normalized by the square of the inflow free-stream velocity. 


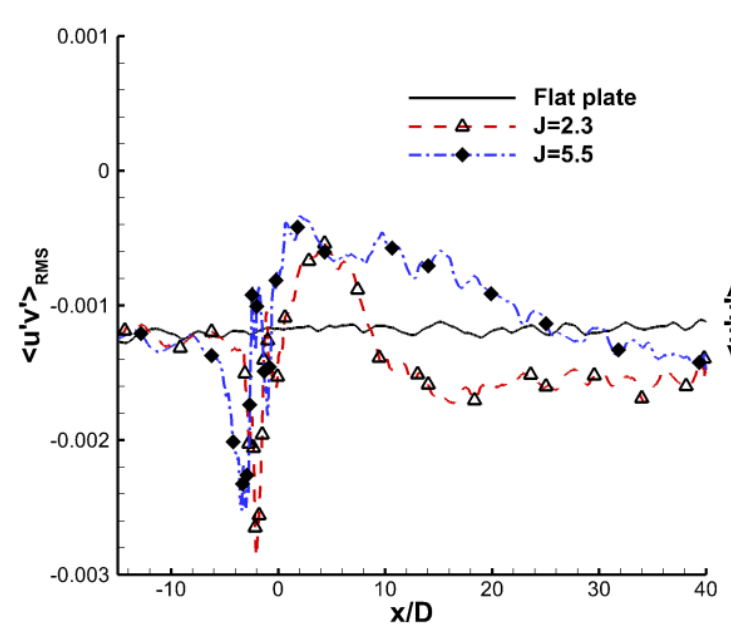

(a) $y / D=0.08$

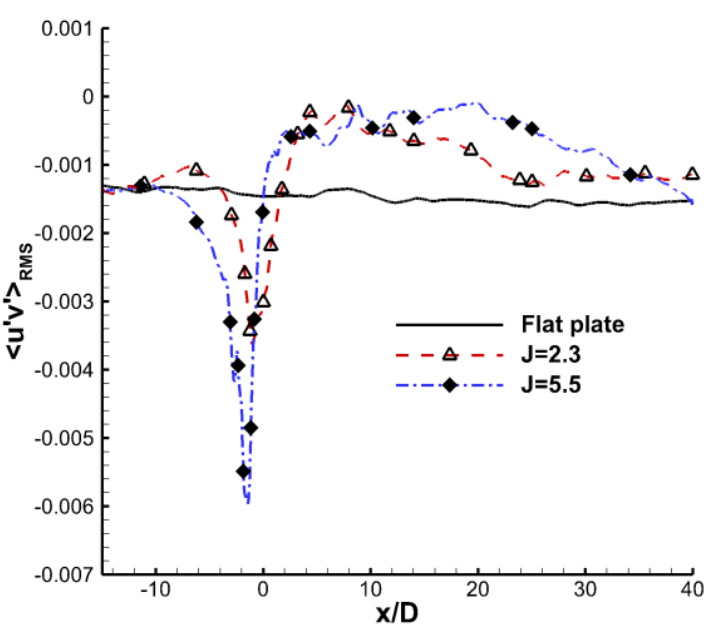

(b) $y / D=0.6$

FIGURE 17. $\left\langle u^{\prime} v^{\prime}\right\rangle$ velocity along the streamwise direction at $y / D=0.08$ and $y / D=0.6$ in the domain $z \in[3.0 D, 3.5 D]$ of different cases, normalized by the square of the inflow free-stream velocity.

\section{Streamline analysis and flow topology}

Figure 18 shows several representative time-averaged streamlines and iso-surface of $M a=0.6$.

The iso-surface of $M a=0.6$ reflects the windward separation bubble ahead of the jet orifice. In figure 18, the streamlines originate from the cross supersonic inflow on the horizontal plane of $y / D=1.0$ upstream of the jet orifice. The streamlines colored by the local wall normal distance demonstrate the motion of the supersonic cross-flow around the jet plume, especially the motion in the wall normal direction. As shown in figure $18(a)$, when the incoming flow is obstructed by the jet plume, the streamlines wrap around the jet orifice and are pushed to higher wall-normal positions and lifted away from the wall by the separation bubble ahead of the jet. Due to the existence of a low pressure region in the jet lee, flow around the jet plume moves from the edge of the separation region to the jet lee. During this process, flow experiences several stages which are well demonstrated by the streamlines. Streamlines firstly move towards the wall from the top of 
the separation bubble when traveling across the separation region, which indicates a flow reattachment from the top of the separation bubble to the wall. During this process, flow experiences an expansion due to the reattachment in a supersonic flow. This expansion is denoted as reattachment expansion, which is consistent with the analysis of the wall pressure shown in figure $9(b)$. After the reattachment, streamlines run further around the jet orifice and keep expanding due to the existence of low pressure zone in the jet lee, as can be seen from the oblique view of figure $18(b)$, where streamlines are colored by the local pressure. Downstream of the jet orifice, streamlines are twisted by the well-known counter-rotating vortex pair (CVP) and concentrate in the jet lee and further rotate downstream to the jet far-field. To summarize, supersonic boundary layer flow around the jet orifice experiences at least two expansions, those are, reattachment expansion and jet lateral expansion. These expansions inevitably change the turbulence status in the supersonic boundary layer.

A representative streamline originating from $y / D=1.0$ on the incoming cross-flow plane is selected from figure $18(b)$ to show the status that the streamline experiences. The pressure and corresponding streamwise pressure gradient along this streamline are given in figure 19(a). The Mach number along the streamline is shown in figure 19(b). Firstly it is seen that the Mach number is always larger than 1.0 along the whole streamline, which demonstrates that the supersonic flow condition is kept in the whole near-field. The negative pressure gradient can be divided into two parts. The first part is a quick expansion region, which corresponds to the reattachment expansion, and the second part is a gradual expansion region, corresponding to the lateral expansion. As has been analyzed in the previous work (Gao et al. 2016), the expansion in supersonic flow leads to a favorable pressure gradient and the reduction of Reynolds shear stress 
induced by the favorable pressure gradient can be analyzed from the Reynolds stress transport equations. After some algebraic manipulations (details can be found in Dussauge \& Gaviglio (1989)) the transport equation for $u_{i}^{\prime} u_{j}^{\prime}$ is obtained as

$$
\frac{D}{D t} u_{i}^{\prime} u_{j}^{\prime}=\frac{\overline{\rho^{\prime} u_{j}^{\prime}} \frac{\partial \bar{p}}{\partial x_{i}}+\overline{\rho^{\prime} u_{i}^{\prime}} \frac{\partial \bar{p}}{\partial x_{j}}}{\bar{\rho}^{2}}+\frac{\overline{u_{i}^{\prime} \frac{\partial p^{\prime}}{\partial x_{j}}}+\overline{u_{j}^{\prime} \frac{\partial p^{\prime}}{\partial x_{i}}}}{\bar{\rho}}
$$

For the mean pressure gradient's contribution on pressure fluctuations (the second term on the right-hand side of equation (1)), the following model by Lumley (1979) is used,

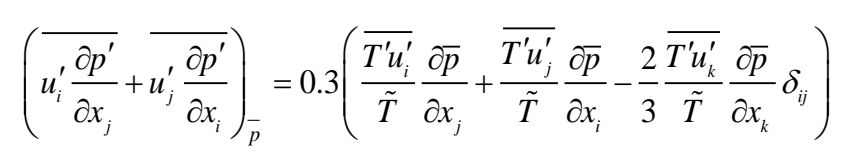

When the flow encounters a streamwise favorable pressure gradient $(\tau \bar{p} / \partial x<0)$ as $\partial \bar{p} / \partial y$ is close to zero near the wall therefore the contribution of the pressure fluctuation term is also negative ( $\delta_{i j}=0$ when $\mathrm{i} \neq \mathrm{j}$ ). Thus the right-hand side of equation (1) is negative. For a steady flow, $D\left(u_{i}^{\prime} u_{j}^{\prime}\right) / D t$ is simplified to $u_{k}\left[\partial\left(u_{i}^{\prime} u_{j}^{\prime}\right) / \partial x_{k}\right]$, which gives the change of the Reynolds shear stresses along a time-averaged streamline. As a consequence, a negative right-hand side of equation (1) indicates that the Reynolds shear stresses decrease in the streamwise direction when the flow travels through a favorable pressure gradient region. As analyzed above, the phenomenon of turbulent coherent structure weakening and local turbulence reduction are all associated with the decrease of Reynolds shear stresses. Based on the numerical results and the above analysis, it is concluded that the double expansion in the supersonic flow leads to the turbulence decay in the lateral boundary layer downstream of the jet.

In figure 20, streamlines passing the horizontal line of $y / D=0.5$ at $x / D=10.0$ in the jet wakes are given. Figure 20 demonstrates the streamline movement in the jet lateral boundary layer. The streamlines running around the jet lateral converge towards the jet leeward centerline. Streamlines 
adjacent to the jet orifice are tilted and folded by the jet leeward 3D flow. It is seen that flow in the jet wake shows strong rotation due to the surface trailing CVP, which is induced by the reattachment flow over the jet lee herringbone separation. This phenomenon has been well analyzed in our previous paper (Sun \& Hu 2018a). The impingement and the surface trailing rotating flow significantly increases the turbulence intensity in the boundary layer, which leads to an increment of turbulence fluctuation downstream of the jet leeward separation and in the far-field. Analysis on TKE and RMS velocities has reflected the turbulence status in the centerline wake. The streaky structures near the centerline wakes in figure 3 are also associated with these interactions. Figure 20 also shows that the streamlines from the cross-flow run through the lateral zone in the vicinity of the jet, which means that the far-field boundary layer flow is related to the near-field lateral region which has a suppressed turbulence status. There is no doubt that the immediate turbulence decay in the jet near-field boundary layer would affect the downstream boundary layer due to the history effect.

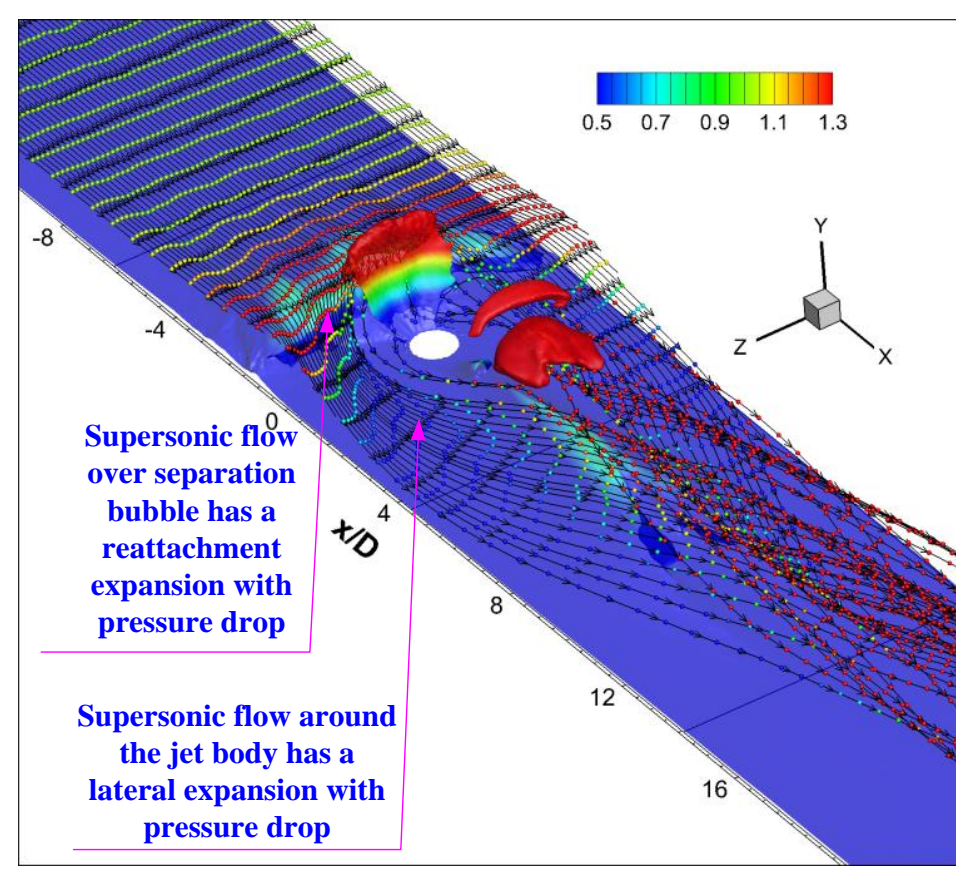


(a) Streamlines originating from plane $y / D=1.0$ in the cross-flows, colored by local wall normal distance

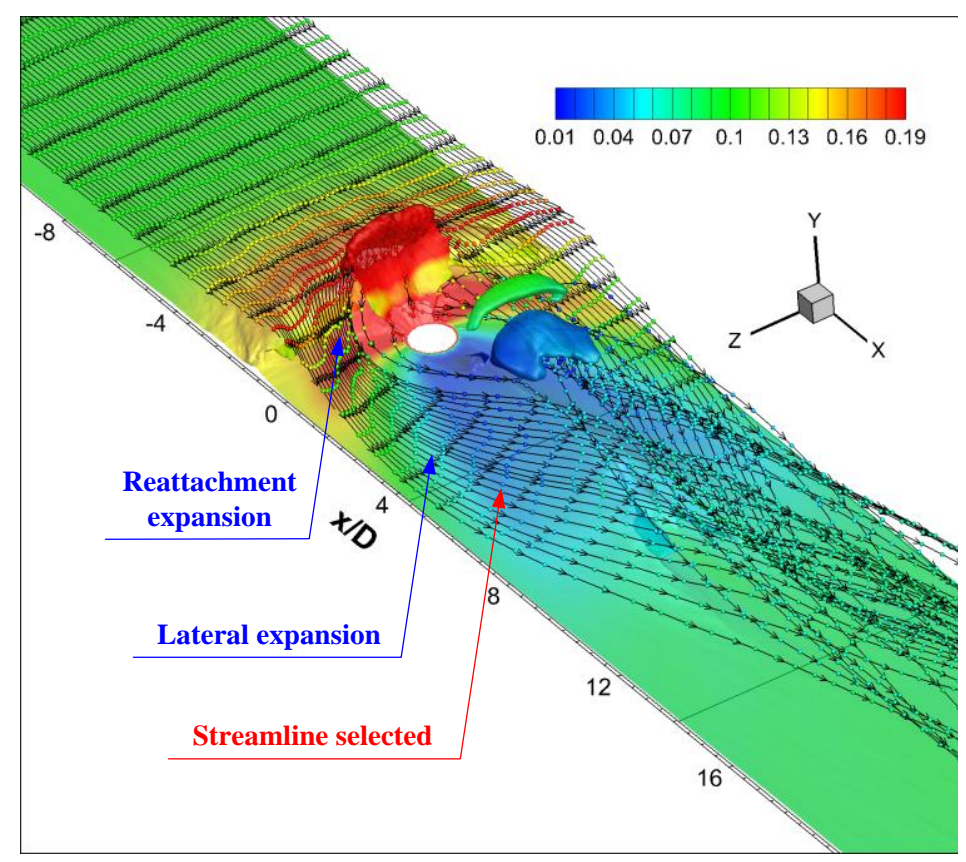

(b) Streamlines originating from plane $y / D=1.0$ in the cross-flows, colored by local pressure

FIGURE 18. Streamlines originating from cross-flow colored by the local wall normal distance (a) and local pressure (b) of the $J=5.5$ case.
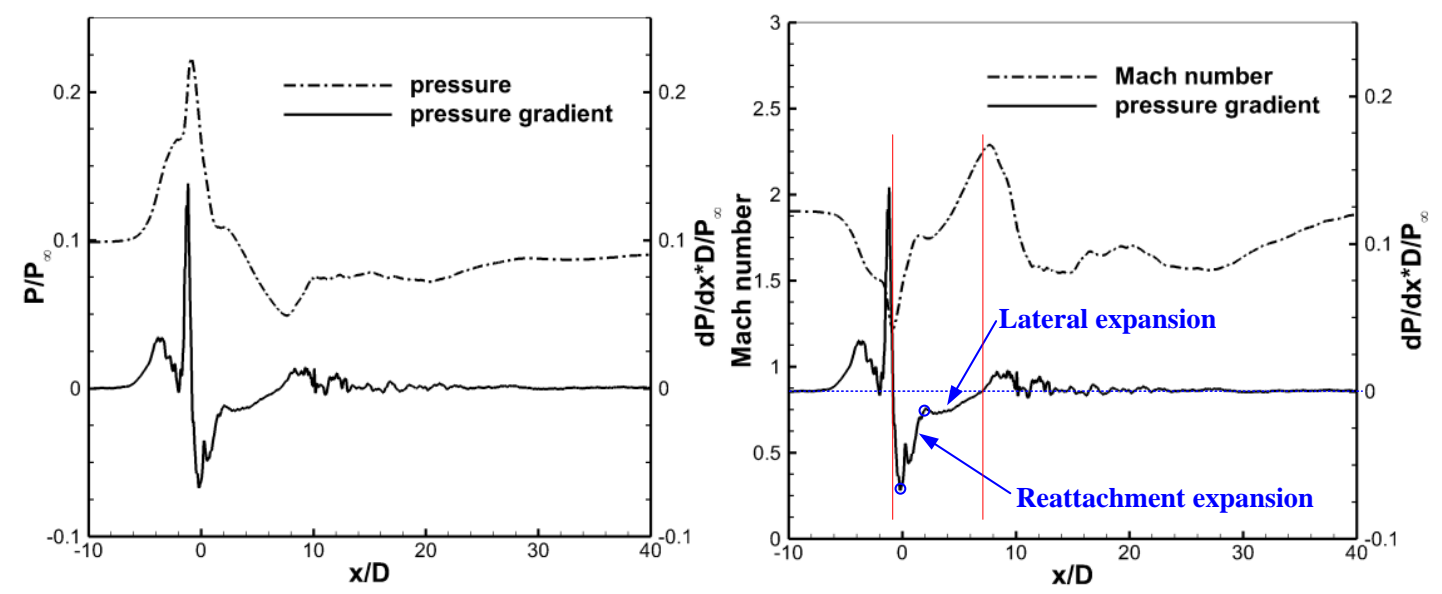

(a) Pressure and pressure gradient distributions (b) Mach number and pressure gradient distributions

FIGURE 19. Pressure, pressure gradient and Mach number distributions along the streamline 
selected in figure $18(b)$ of the $J=5.5$ case.

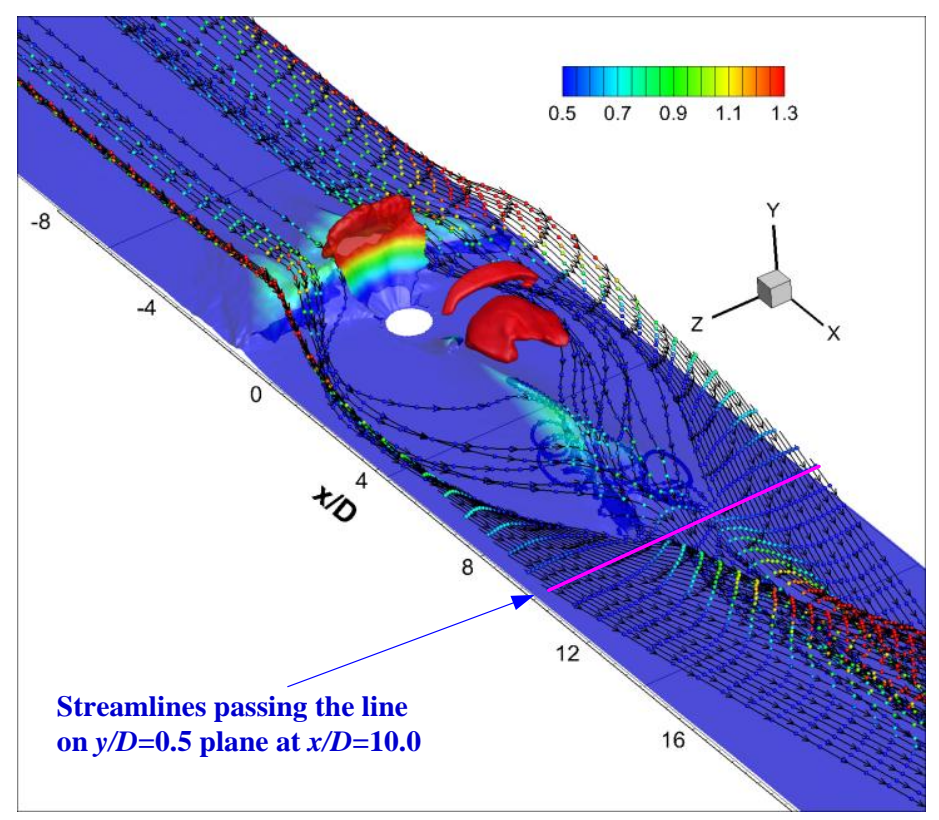

(a) Streamlines passing through a line at $x / D=10.0$ and $y / D=0.5$ in the jet wake flow, colored by local wall normal distance

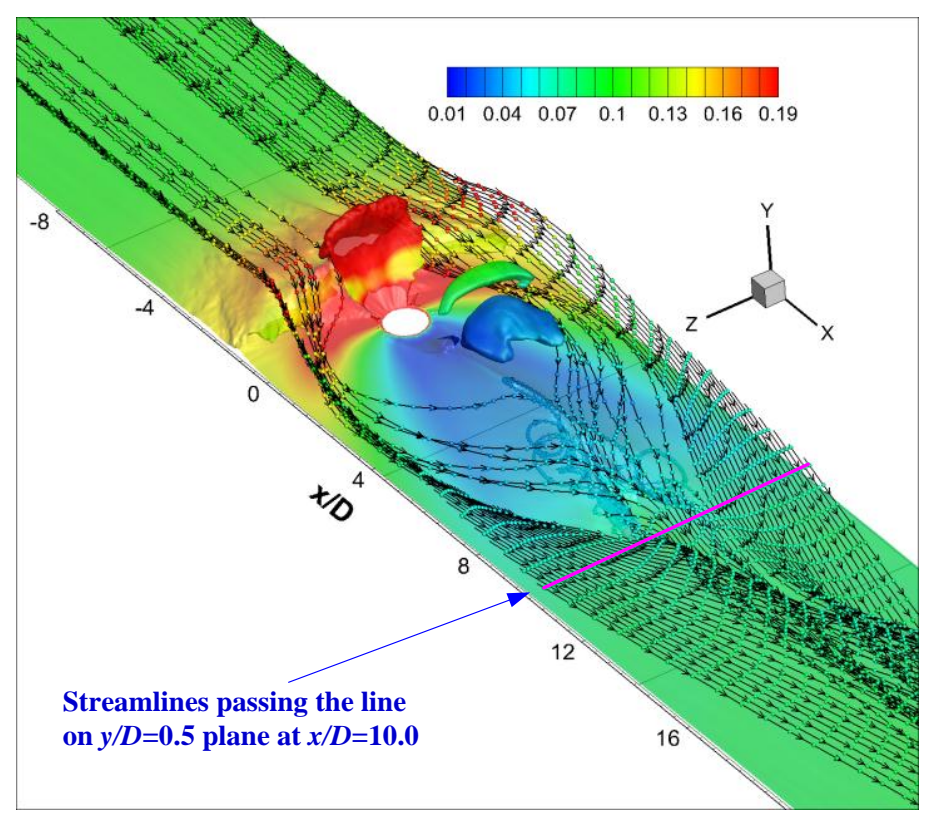

(b) Streamlines passing through a line at $x / D=10.0$ and $y / D=0.5$ in the jet wake flow, colored by

$$
\text { local pressure }
$$

FIGURE 20. Streamlines originating from jet wake flow colored by the local wall normal distance

(a) and local pressure (b) of the $J=5.5$ case. 
Figure 21 gives an oblique-view and a top-view of the double expansion with iso-surfaces of $M a=0.6$ and 2.9 superimposed by pressure contours, which demonstrates that the supersonic boundary layer flow experiences double expansion around the sonic jet which leads to turbulence decay in the vicinity of the jet lateral. The first expansion corresponds to the reattachment expansion. The streamlines go across the windward separation bubble ahead of the jet and reattach to the wall, experiencing an expansion in the supersonic flow. The second expansion represents the lateral expansion occurs in the lateral near to the jet orifice. As shown in figure 21(a), the barrel shock detaches from the wall surface and creates the low-pressure region which absorbs the lateral flow, which was well analyzed in our previous work (Sun \& Hu 2018b). The streamlines follow the lateral side of the barrel shock and run into the low-pressure region behind the jet, experiencing a lateral expansion in the supersonic flow. The double expansion leads to the apparent turbulence decay in the lateral of the jet. The streamlines from the cross-flow run through this lateral region (as shown in figure 20) and suffer from the suppressed turbulence status, which results in the slow recovery of the outer boundary layer in the far-field. 


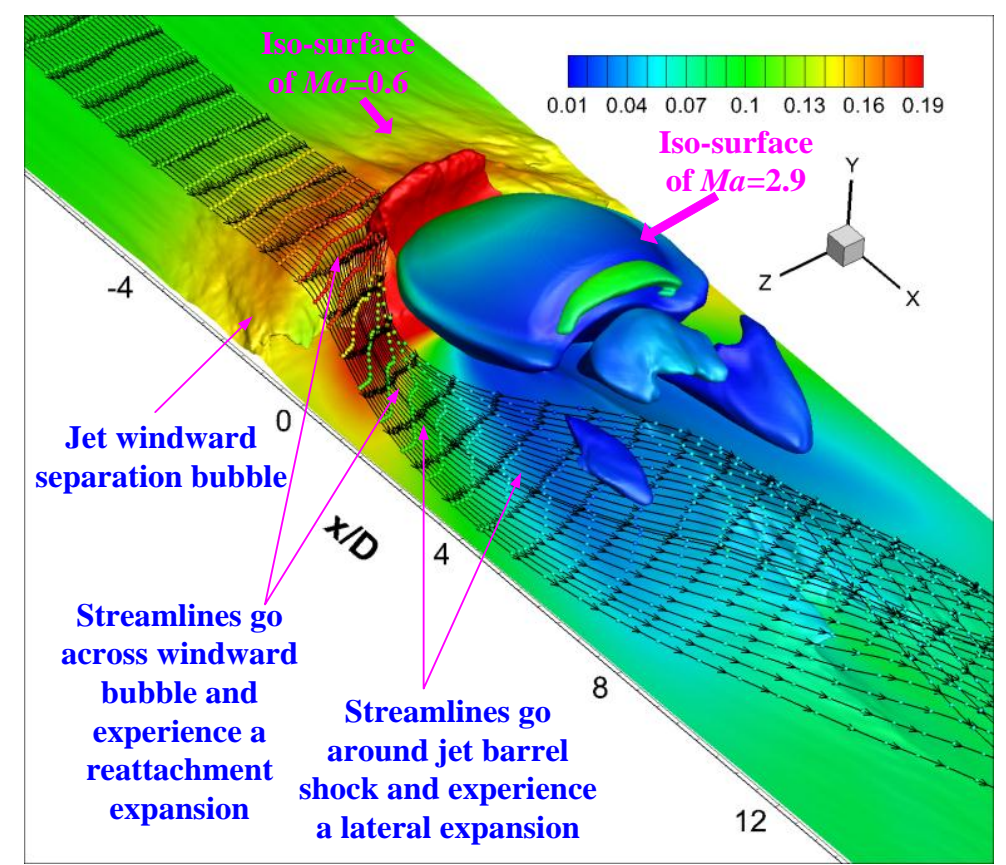

(a) An oblique view of the streamlines with iso-surfaces of $M a=0.6$ and $M a=2.9$, superimposed by

$$
\text { pressure contours }
$$

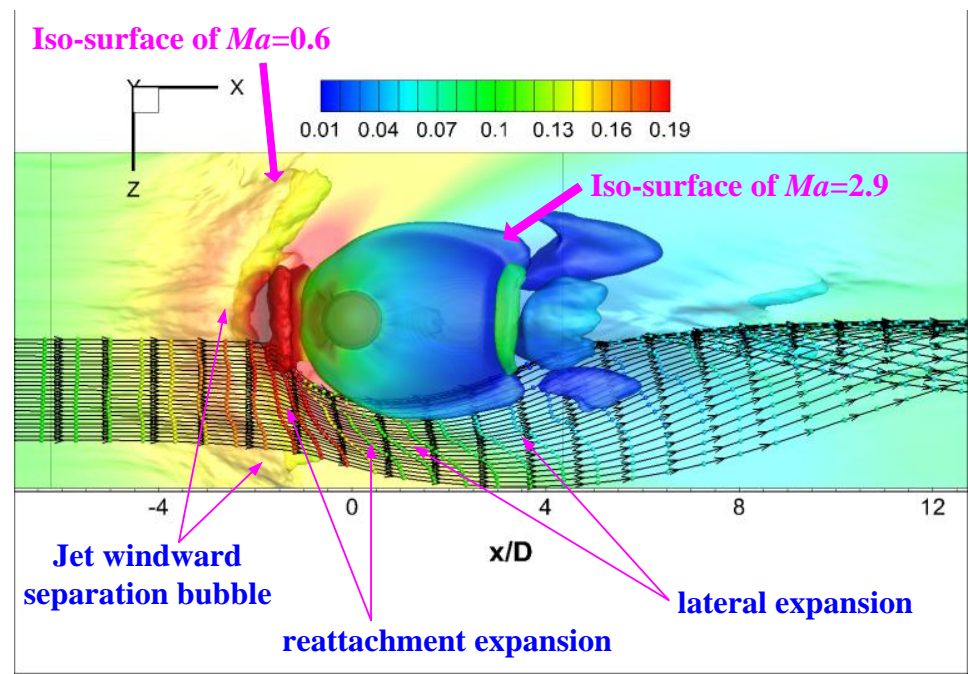

(b) A top view of the streamlines with transparent iso-surfaces of $M a=0.6$ and $M a=2.9$,

superimposed by pressure contours

FIGURE 21. The double expansion for a supersonic boundary layer flow around the jet of the $J=5.5$ case, superimposed by pressure contours.

\section{Preliminary experiments to confirm the turbulence decay phenomenon}


All the results of these activities are described in Appendix A-B. The experiments are conducted to investigate the supersonic turbulent boundary layer with a transverse jet by using nano-particle laser scattering (NPLS) technique. The instantaneous images and the averaged data from NPLS images both confirm the existence of the turbulence decay phenomena in reality. The experiment validation is preliminary and indirect since there are several reasons. Firstly, there is no particle fed in the injection. The dark color in the jet regime probably represents no particle, which does not reflect the density variation in the flow field. The jet plume expands and extends in the spanwise zone, which means that the density field reflected by the NPLS image is polluted by the jet injectants and gives invalid density variation and inaccurate turbulence status. According to the NPLS images shown in figure 24 , the spanwise zone $z / D \in[-5,5]$ near to the jet orifice is contaminated by the non-particle flow and the local turbulence status is unknown. This means that the near-field zone $z / D \in[-3.5,3.5]$ we are interested in can not be well revealed. The comparison between the simulation and the experiment can not be made directly. This problem is difficult to solve. Even though we conduct PIV (particle image velocimetry) test to obtain the velocity field, there are little particles in the near-field zone and it is impossible to calculate the accurate velocity. We can only observe the turbulence status in the lateral boundary layer which has a longer enough distance away from the jet orifice and in the far-field boundary layer where the cross-flow has carried enough particles to reflect the density variation. Secondly, the density variation does not correspond to the turbulent velocity fluctuation directly. As Martin (2007) pointed out, even assuming the pressure fluctuations are negligible in a turbulent boundary layer, the strong Reynolds analogy gives

$$
\frac{\rho^{\prime}}{\bar{\rho}}=(\gamma-1) M a^{2} \frac{u^{\prime}}{\bar{u}}
$$


which means $\frac{\rho^{\prime}}{\bar{\rho}}$ is not proportional to $\frac{u^{\prime}}{\bar{u}}$ since local Mach number still needs to be determined. Thus the density fluctuation obtained from NPLS images is only the indirect evidence for turbulence. Thirdly, the NPLS experiment only acquires certain transverse and longitudinal two-dimensional slices of the flow field. The three dimensional configuration of the jet plume and its interaction with the supersonic cross-flow is difficult to be revealed by the slices. The turbulence status inferred from the NPLS images is not easily related to the turbulence change near the jet.

Anyway, the current NPLS experiments basically demonstrate the turbulence suppression caused by the jet in the supersonic cross-flow. Meanwhile the experiment verification is far away from a complete or thorough validation. The current simulations and the experiment provide a challenging and open topic for other researchers. Any possible advanced optical technique could be tested for this complex flow phenomenon, especially if the near-wall region in the vicinity of the jet orifice could be well measured. As discussed above, this is very hard.

\section{Conclusions}

In the present study, direct numerical simulations are conducted to uncover physical aspects of a transverse sonic jet injected into a supersonic cross-flow at Mach number 2.7, focusing on the supersonic boundary layer flow characteristics subjected to the sonic jet. Simulations are run for two different jet-to-cross-flow momentum flux ratios of 2.3 and 5.5. A flat plate boundary layer without jet $(J=0.0)$ is simulated for comparison.

The simulation provides the instantaneous and averaged flow features in the supersonic boundary layer with the sonic jet injection. It is found that in the near-wall flow, favorable pressure gradients exist and turbulent coherent structures are weakened significantly in the vicinity 
around the jet and the status retains in the downstream recovery region on the lateral of the jet. The boundary layer thickness decreases significantly with an increased $J$ in the lateral downstream of the jet. Turbulence in the region near the jet leeward centerline is amplified due to the collision of the cross-flow running around the jet and its interaction with the boundary layer.

A characteristic reduction in TKE and shear stress profiles in the boundary layer along the streamwise direction in the lateral near-wall region is exhibited and compared to the flat plate case. The shear stress decreases significantly and the turbulence in the outer layer of the supersonic boundary layer decays sharply and is consistently suppressed along the streamwise direction in the lateral. The decay blocks the exchange of energy between the inner layer and the mainstream, and leads to a quick recovery in the inner boundary layer and a slow recovery in the outer layer. In the far-field boundary layer, TKE is higher in the jet centerline than the lateral along the span. Higher jet momentum flux ratio leads to a more intense turbulence suppression in the lateral downstream.

Analysis of cross-flow streamlines exhibits a double expansion character around the sonic jet which leads to turbulence decay in the vicinity of the jet lateral. The first expansion is the reattachment expansion, which occurs in the reattachment region downstream of the windward separation bubble induced by the jet. The second expansion is the lateral expansion, which occurs in the lateral side of the barrel shock. The double expansion leads to the turbulence reduction and the weakening of the coherent structures in the jet near-field and affects the turbulence in the far-field boundary layer due to the history effects.

A preliminary experiment based on nano-particle laser scattering technique is conducted for $J=5.5$. The instantaneous experimental results reveal that the boundary layer thickness in the lateral region downstream of the jet decreases and also reflect the local turbulence reduction. From 
an overall view of the averaged and RMS data of the NPLS images, it is found that the intensity of the density fluctuation decreases in the jet lateral side, which basically confirms the existence of turbulence decay phenomenon in the near-wall lateral flow downstream of the jet.

\section{Acknowledgement}

Sincere thanks to Professor N. D. Sandham of the University of Southampton for providing the SBLI DNS code and the helpful discussion on data analysis.

This work is funded by the National Science Foundation of China (grants: 11472305 and 11522222) and outstanding youth fund of National University of Defense Technology.

Supercomputer time provided by the UK Engineering and Physical Sciences Research Council under the project 'Thermal and Reactive Flow Simulation on High-End Computers' (Grant No. $\mathrm{EP} / \mathrm{J} 016381 / 1)$ is gratefully acknowledged.

\section{Appendix A}

This paper uses Nano-particle-based Planar Laser Scattering technology (developed by Zhao et al. (2009)) to conduct the experiments. The NPLS system is composed of a double-pulsed Nd:YAG laser with wavelength of $532 \mathrm{~nm}$ and pulse energy of $520 \mathrm{~mJ}$ per $6 \mathrm{~ns}$, a IMPERX charge-couple device $(\mathrm{CCD})$ camera of $4000 \times 2672$ pixel $^{2}$, a nano-particle generator, a computer and a synchronizer. The nano-particle are seeded in the supersonic flow and acts as the tracer and its relaxation time is $66.3 \mathrm{~ns}$ and effective diameter is $42.5 \mathrm{~nm}$, as given by Zhao et al. (2009). It is reasonable that the nano-particles follow the fluctuation of small scale turbulence in supersonic flows (Sun et al 2013; Wang \& Wang 2016). The maximum resolution of the system reaches 
$10 \mu \mathrm{m} /$ pixel. Experiments are conducted in a Mach 2.7 suction-type supersonic wind tunnel. The test section of the wind tunnel is $400 \mathrm{~mm}$ long with the cross-sectional size of $200 \times 200 \mathrm{~mm}^{2}$. Test models are mounted horizontally in the test section. The model is a flat plat with a circular injector (diameter $D$ is $2 \mathrm{~mm}$ ), as shown in figure 22. Assume the origin of the coordinate system lies on the center of the orifice, resolution on slice $z=0 \mathrm{~mm}$ and $z=10 \mathrm{~mm}(z / D=5.0)$ is $0.0259 \mathrm{~mm} /$ pixel for a side-view visualization, and resolution on slice $y=2 \mathrm{~mm}(y / D=1.0)$ and slice $y=4 \mathrm{~mm}(y / D=2.0)$ is $0.0332 \mathrm{~mm} / \mathrm{pixel}$ and $0.0344 \mathrm{~mm} /$ pixel for a top-view visualization respectively.

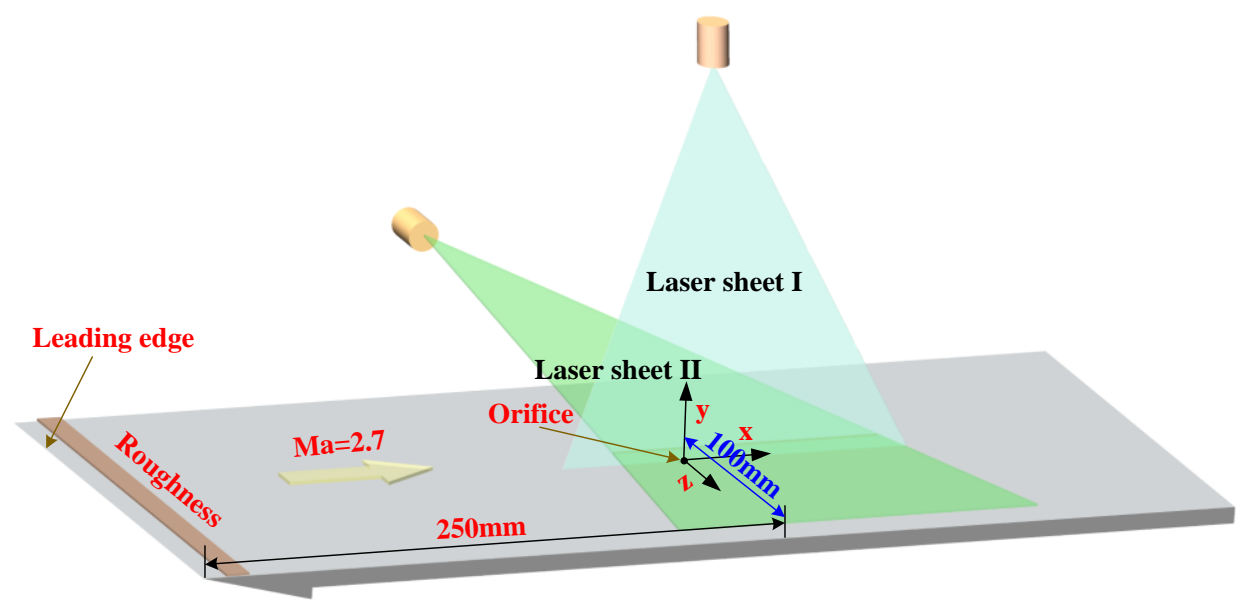

FIGURE 22. Schematic of the experimental setup

As shown in figure 22 , a roughness belt with size of $10 \times 190 \mathrm{~mm}^{2}$ is placed $10 \mathrm{~mm}$ downstream of the leading edge to shift the inflow boundary layer from laminar to turbulent state. The method can be referenced in Wang \& Wang (2016). To ensure the boundary layer is fully developed, a long flat plate with length $250 \mathrm{~mm}$ is set upstream of the jet orifice. The $x$-axis is set along the centerline of the flat plate.

The air inflow parameters are set in accordance with the $M a=2.7$ with stagnation pressure $P_{0}$ $=101325 \mathrm{~Pa}$, stagnation temperature $T_{0}=300 \mathrm{~K}$ with the corresponding unit Reynolds number is $\operatorname{Re}_{l}=8.96 \times 10^{6}$. The high spatiotemporal resolution NPLS image of the incoming turbulent 
boundary layer is given in figure 23 , from which the coherent structures in the inner part and the outer part of the turbulent boundary layer could be distinguished. The velocity data in the wall boundary-layer at $x=-10 \mathrm{~mm}$ from PIV measured field and a theoretical prediction described as Touber (2010) is compared. From the comparison the $99 \%$ boundary-layer thickness is estimated as $\delta=6.0 \mathrm{~mm}$, which is basically consistent with the current simulation.

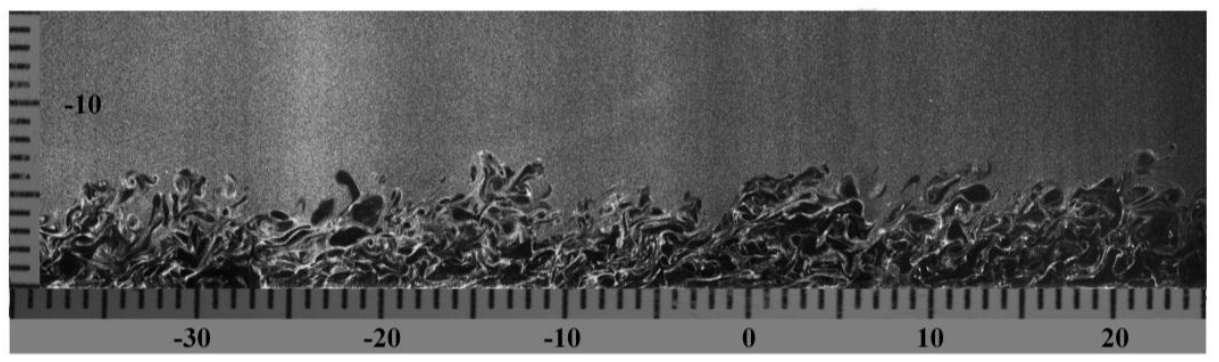

FIGURE 23. The flat plat boundary layer without the jet imaged by NPLS

Nitrogen gas injection without nano-particles are used and the jet properties are set to correspond to the injection parameters as shown in Table 3. The stagnation is higher than the setup in the simulation since the jet orifice has a low coefficient of the mass flow rate about 0.86 . The given stagnation pressure assures that $J$ is basically equal to 5.5 .

TABLE 3. Nitrogen jet condition for the experiment

\begin{tabular}{lcccc}
\hline Jet-to-cross-flow & Orifice & Mach & Stagnation & Stagnation \\
momentum $J$ & diameter $D$ & number $M_{j}$ & temperature $T_{0 j}$ & pressure $P_{0 j}$ \\
5.5 & $2 \mathrm{~mm}$ & 1.0 & $300 \mathrm{~K}$ & $388 \mathrm{kPa}$ \\
\hline
\end{tabular}

\section{Appendix B}

Figure 24 gives the representative NPLS images of the instantaneous streamwise flow structures with the sonic jet into the $\mathrm{Ma}=2.7$ supersonic cross-flow. The large-scale structures, the 
bow shock, the separated shock, and the boundary layer upstream of the jet can be observed in figure $24(a)-(b)$. Chaotic turbulent structures in the boundary layer upstream of the jet can be well identified in figure $24(c)-(d)$. The boundary layer downstream of the orifice is also visible in the figure $24(b)$. It is seen that the boundary layer downstream of the jet orifice is affected by the jet plume and the boundary layer thickness could be identified to decrease in the far-field compared to the upstream boundary layer. This phenomenon agrees well with the numerical prediction in figure 8. Since the nitrogen jet carries no nano-particle, the coherent structures in the wake flow displayed by the nano-particles in figure $24(c)-(d)$ come from the cross-flow. It is found that the jet interacts with the upcoming boundary layer significantly. The turbulent structures crush into the separation region upstream of the jet. In the lateral of the jet, the structures of the horseshoe vortex which originates from the upstream separation induced by the bow shock are identified. Downstream of the separation region, it is seen that the cross-flow runs around the jet and impinges into trailing wake region.

In the vicinity downstream of the jet leeward separation, the wake structures in dark color cannot be well identified since the flow might originate from the non-particle jet. At $y / D=1.0$ $(y=2 \mathrm{~mm})$ slice, it is identified that the intensity of the turbulence in the lateral side downstream of the jet orifice is lower than the flow upstream of the jet in the boundary layer, as enclosed in the red ellipse. The phenomena reveal two characters in the lateral boundary layer. The first is that the boundary layer thickness in the lateral downstream of the jet decreases, which is consistent with the result in figure $24(b)$. This is due to the quick reattachment of the supersonic flow downstream of the windward separation, as indicated by the simulation. The second is that the turbulence in the jet lateral is suppressed. In the centre downstream of the jet wake, the grey coherent structures 
occurs in the jet lee, which represents that the cross-flow runs around the jet and concentrates in the jet lee and leads to a local turbulence amplification. The turbulent coherent structures downstream of the jet wake extend in the spanwise zone and affect the lateral boundary layer. The effect of the wake turbulence is also an important factor for the turbulence recovery of the lateral boundary layer from the suppression near to the jet.

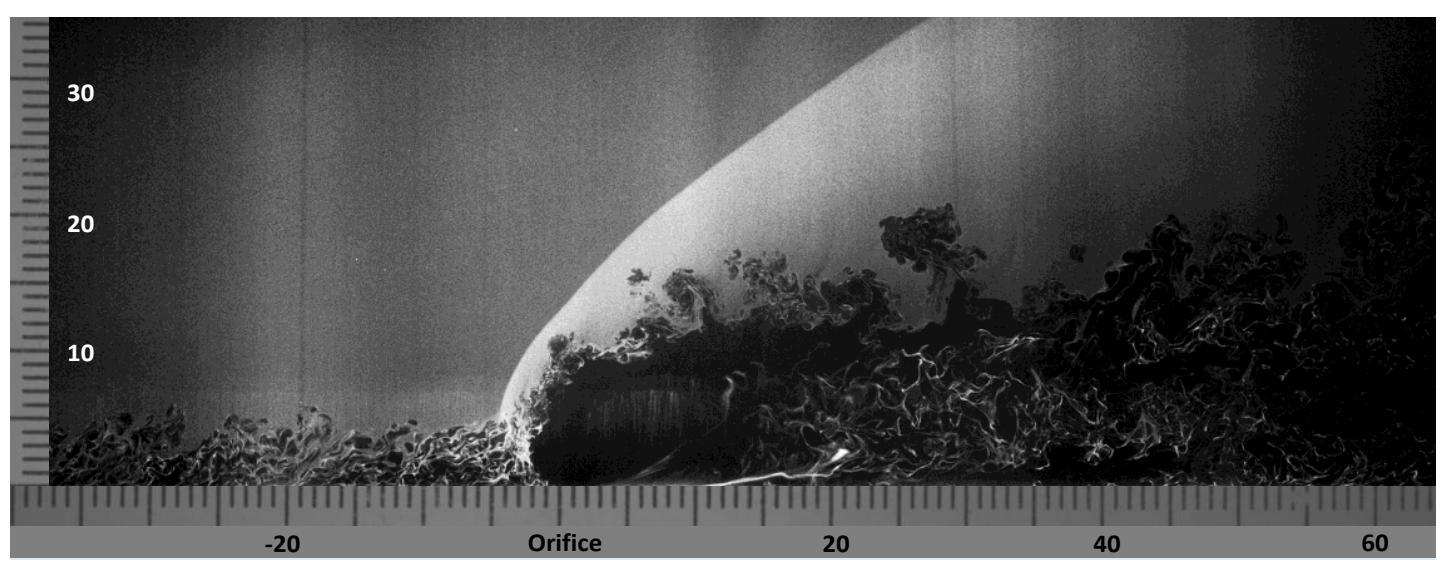

(a) $z / D=0.0(z=0 \mathrm{~mm})$

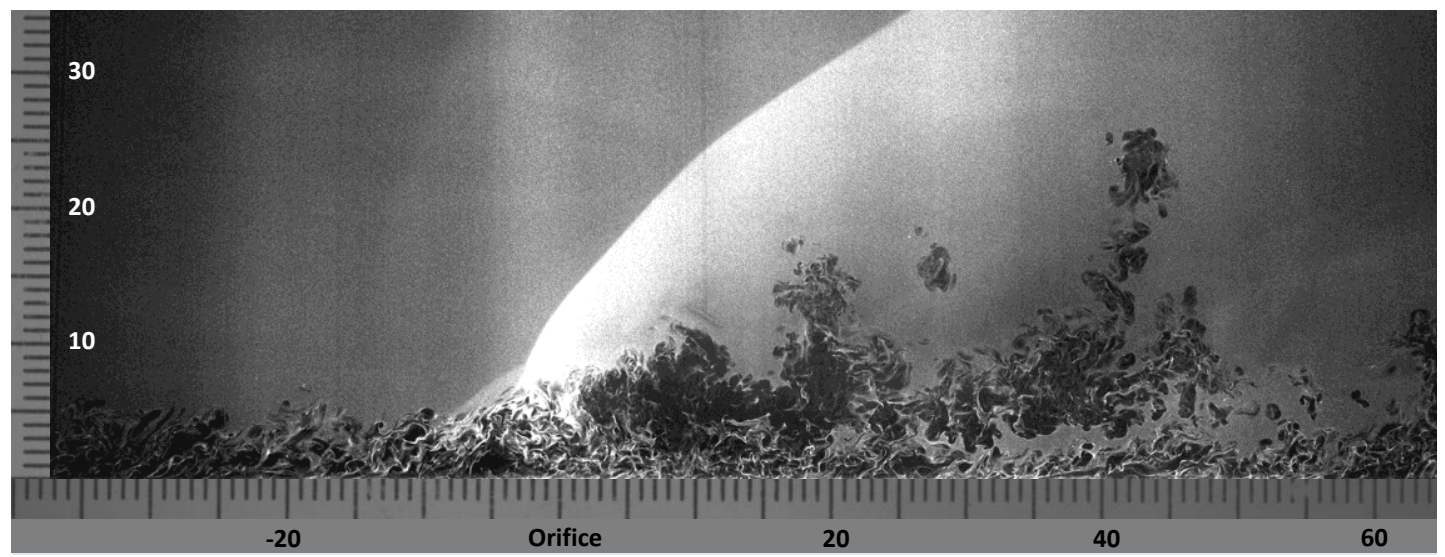

(b) $z / D=5.0(z=10 \mathrm{~mm})$ 


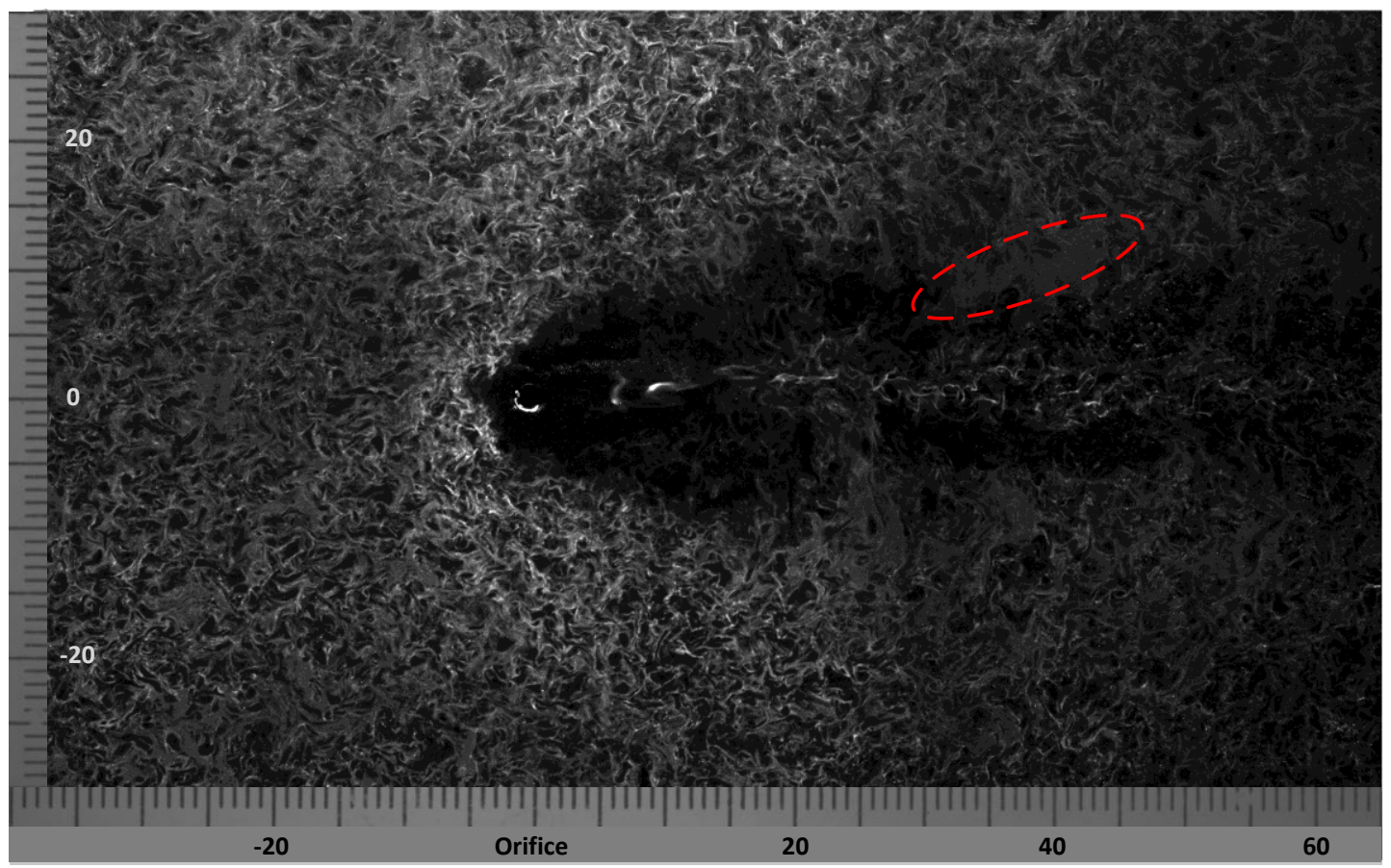

(c) $y / D=1.0(y=2 \mathrm{~mm})$

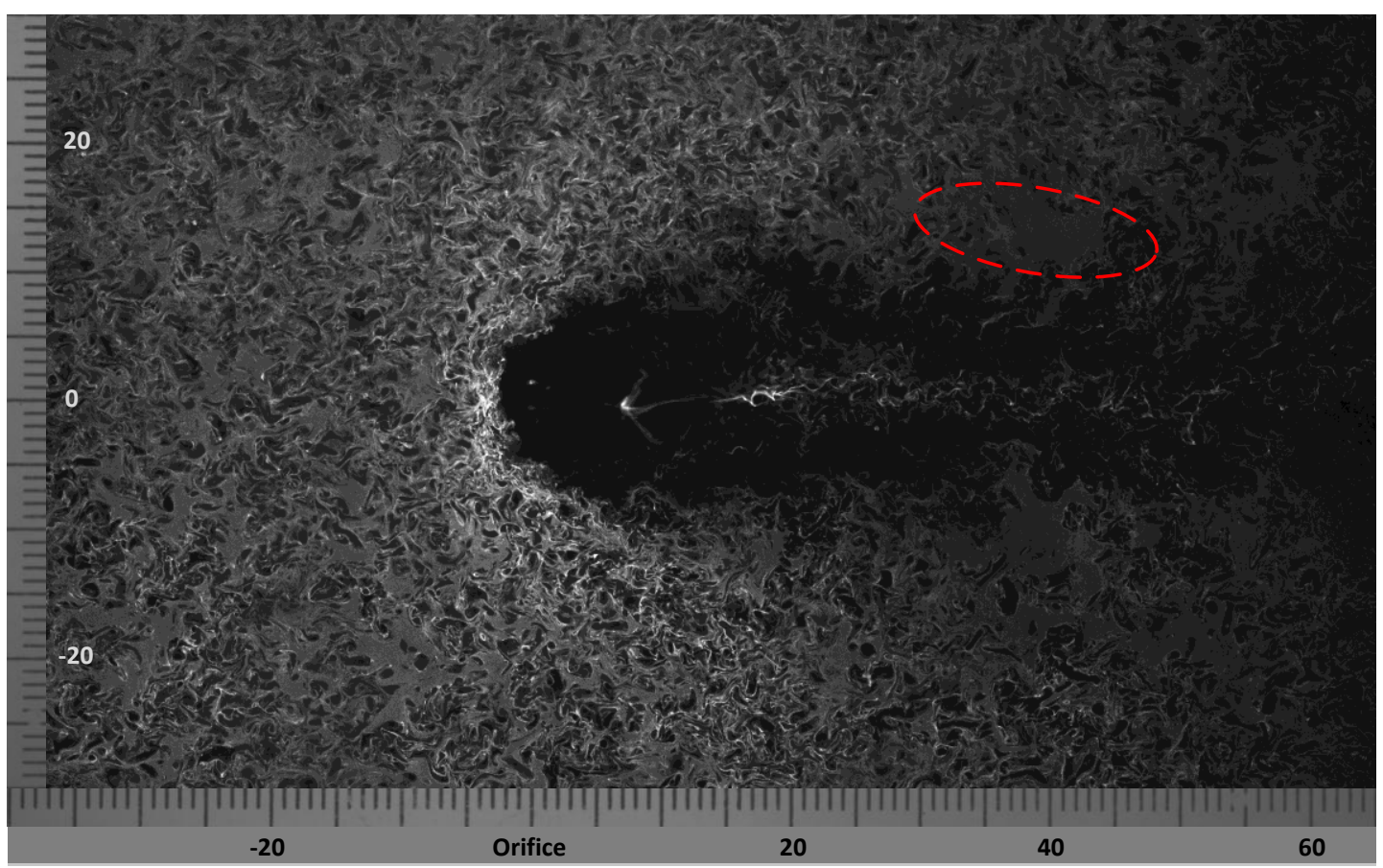

(d) $y / D=2.0(y=4 \mathrm{~mm})$

FIGURE 24. Instantaneous streamwise flow structures of the sonic jet into the supersonic cross-flow with different slices of the flow-field 

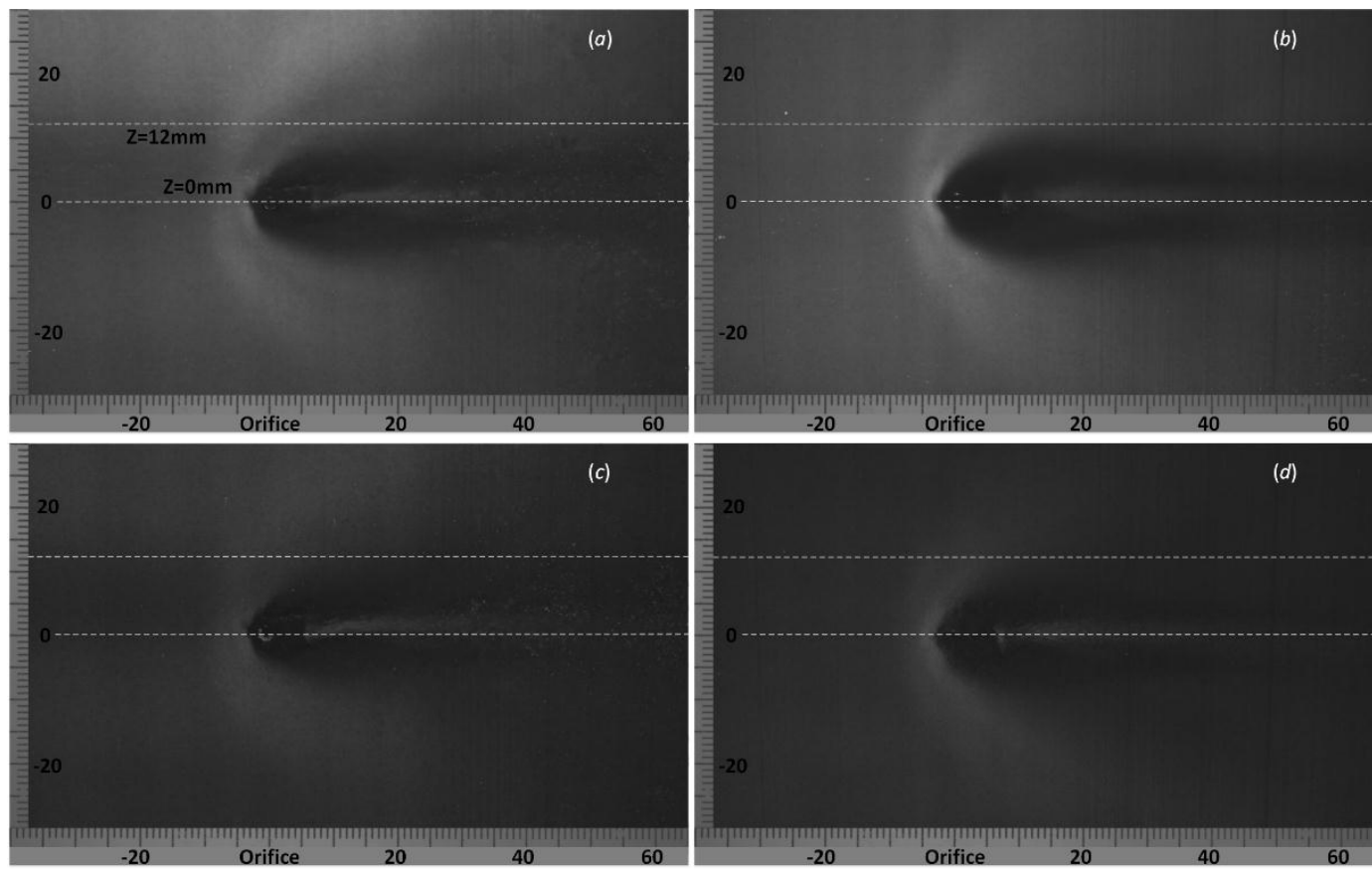

(a) Averaged NPLS images on $y / D=1.0$

(b) Averaged NPLS images on $y / D=2.0$

(c) RMS of NPLS images on $y / D=1.0$

(d) RMS of NPLS images on $y / D=2.0$

FIGURE 25. Averaged and RMS streamwise flow structures of the sonic jet into the supersonic cross-flow on $y / D=1.0$ and 2.0 slices. $z / D=0.0(z=0 \mathrm{~mm})$ and $z / D=6.0(z=12 \mathrm{~mm})$ lines on the slices are selected.

Averaged and RMS of the NPLS images are calculated and shown in figure 25. The color of NPLS image reflects the density $(\rho)$ change on the slice, as concluded by Zhao et al. (2009). In the jet wake it clearly shows that an impingement occurs in the jet leeward and the V-shape wake exist. RMS images reflect the local density fluctuation, as denoted as $\rho_{R M S}$ and averaged images reflect the local density intensity $\bar{\rho}$. In the vicinity of the jet orifice, it is hard to evaluate the local density fluctuation since there are no particles in the injectants. That is why we can not give the density fluctuation in the domain of $-3.5<z / D<3.5$ directly to make comparison with the simulated data. In the lateral side, the zone of $z / D>5.0$ could be treated as the non-polluted region from the injectants. It is recognized that the local density fluctuation in the zone $z / D>5.0$ of the lateral boundary layer is clearly revealed. The density fluctuation in the jet wakes in the centre downstream of the jet 
with $x / D>5.0$ is also clearly revealed.

The location at $z / D=6.0$ has a long enough distance away from the jet center, which ensures that the location is not affected by the non-particle flow from the jet. Figure 26 gives $\rho_{R M S} / \bar{\rho}$ distribution along $z / D=6.0$ and $z / D=0.0$ at $y / D=1.0$ and $y / D=2.0$ corresponding to figure 25 . In figure 26(a), the peak value of $\rho_{R M S} / \bar{\rho}$ corresponds to the jet windward separation. The magnitude of $\rho_{R M S} / \bar{\rho}$ at $y / D=1.0$ starts to decrease when the flow goes across the separation region. From figure 26, we can see that $\rho_{R M S} / \bar{\rho}$ at $z / D=6.0$ decreases to a minimum value at nearly $x / D=20.0(x=40 \mathrm{~mm})$, indicating that local density fluctuation is reasonably suppressed. A careful observation shows that the minimum $\rho_{R M S} / \bar{\rho}$ intensity in the boundary layer is reduced about $9.8 \%$ of the intensity in the upstream boundary layer. In the downstream region of $x / D=20.0$, $\rho_{R M S} / \bar{\rho}$ starts to increase, which reflects a local density fluctuation recovery due to the development of the lateral boundary layer and the spanwise extension of the wake flow. At $y / D=2.0$ the phenomenon is lagged since at this height the local boundary layer thickness decreases and it takes a longer distance to get recovered when compared to $y / D=1.0$. At $z / D=0.0$, the magnitude of the density fluctuation in the flow downstream of the orifice is higher than that upstream of the orifice, which can be attributed to the strong flow interaction by the collision of the cross-flows. In the zone of $-5.0<x / D<5.0$ due to the non-particle flow related to the jet, the density fluctuation is not valid in figure $26(b)$.

This experiment basically confirms the existence of the turbulence delay phenomenon in the lateral supersonic boundary layer around the jet orifice, and reveals the turbulence amplification in the jet wake near-wall flow. 


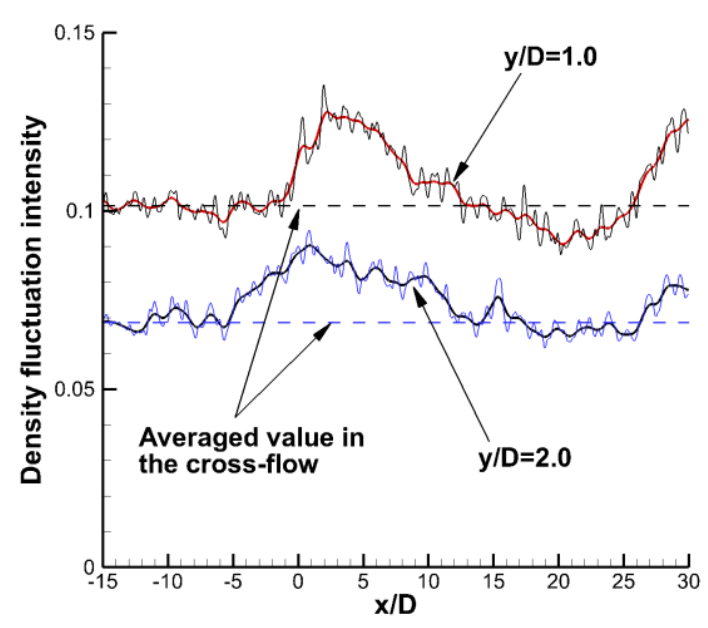

(a) $z / D=6.0$

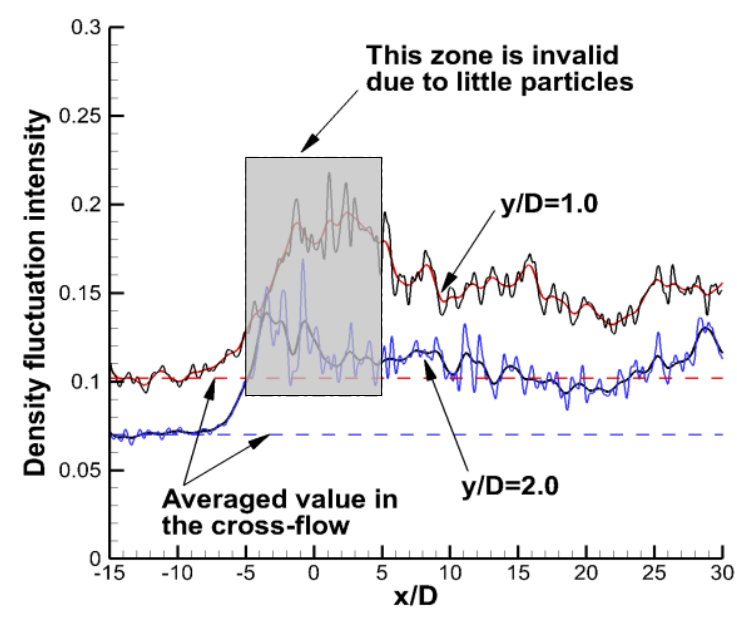

(b) $z / D=0.0$

FIGURE 26. Density fluctuation intensity at $z / D=6.0(z=12 \mathrm{~mm})$ and $z / D=0.0(z=0 \mathrm{~mm})$ on $y / D=1.0$ $(y=2 \mathrm{~mm}), y / D=2.0(y=4 \mathrm{~mm})$ slice respectively. The smoothed line by averaging 1000 points for the original data is superimposed.

\section{Reference}

Arnette S, Samimy M, Elliott G. The effects of expansion on the turbulence structure of a compressible boundary layer. J. Fluid. Mech., 1998, 367:67-105

Ben-Yakar A, Mungal G M, Hanson R K. Time evolution and mixing characteristics of hydrogen and ethylene transverse jets in supersonic crossflows. Phys. Fluids, 2006, 18:26-101

Chai X, Iyer P S, Mahesh K. Numerical study of high speed jets in crossflow. J. Fluid Mech. , 2015, $785: 152-188$

Dickmann D A, Lu F K. Shock/Boundary-Layer Interaction Effects on Transverse Jets in Crossflow over a Flat Plate. J. Spacecr. Rockets, 2009, 46 (6):1132-1141

Dussauge J, Gaviglio J. The rapid expansion of a supersonic turbulent flow: role of bulkdilatation. J.

Fluid. Mech., 1987, 174:81-112

Everett D E, Woodmansee M A, Dutton J C, Morris M J. Wall Pressure Measurements for a Sonic Jet 
Injected Transversely Into a Supersonic Crossflow. J. Propul. Power, 1998, 14 (6):861-886

Gamba M, Mungal M G. Ignition, flame structure and near-wall burning in transverse hydrogen jets in supersonic crossflow. J. Fluid Mech. , 2015, 780:226-273

Gao T Y, Liang J H, Sun M B, Zhao Y H. Analysis of Separation Modes Variation in a Scramjet Combustor with Single-Side Expansion. AIAA J., 2017, 55 (2):563-578

Gruber M R, Nejad A S, Chen T H, Dutton J C. Large structure convection velocity measurements in compressible transverse injection flowfields. Exp. Fluids, 1997, 22:397-407

Karagozian A R. Transverse jets and their control. Prog. Energy Combust. Sci. , 2010, 36 (5):531-553

Kawai S, Lele S K. Large-Eddy Simulation of Jet Mixing in Supersonic Crossflows. AIAA J., 2010, 48

(9):2063-2083

Liang C H, Sun M B, Liu Y, Yang Y X. Shock wave structures in the wake of sonic transverse jet into a supersonic crossflow. Acta Astronaut., 2018, 148:12-21

Lumley J L. Computational Modeling of Turbulent Flows. Advances in Applied Mechanics, 1979, 18: 123-176

Mahesh K. The interaction of jets with crossflow. Annu. Rev. Fluid Mech. , 2013, 45:379-407

Martin M. Direct numerical simulation of hypersonic turbulent boundary layers. Part 1. Initialization and comparison with experiments. J. Fluid Mech., 2007, 570 (347-364)

Portz R, Segal C. Penetration of Gaseous Jets In Supersonic Flows. AIAA J., 2006, 44 (10):2426-2429

Rana Z A, Thornber B, Drikakis D. Transverse jet injection into a supersonic turbulent cross-flow. Phys. Fluids, 2011, 23 (046103):1-22

Sandham N D. Effects of Compressibility and Shock-Wave Interactions on Turbulent Shear Flows.

Flow Turbul. Combust., 2016, 97:1-25 
Sandham N D, Johnstone R, Jacobs C T. Surface-sampled simulations of turbulent flow at high Reynolds number. Int J Numer Meth Fluids, 2017:1-13

Sandham N D, Li Q, Yee H C. Entropy splitting for high-order numercial simulation of compressible turbulence. J. Comput. Phys., 2002, 178:307-322

Sandham N D, Schülein E, Wagner A, Willems S, Steelant J. Transitional shock-wave/boundary-layer interactions in hypersonic flow. J. Fluid Mech., 2014, 752:349-382

Santiago J G, Dutton J C. Velocity measurements of a jet injected into a supersonic crossflow. J. Propul. Power 1997, 13 (2):264-273

Schetz J, Billig F S. Penetration of gaseous jets injected into a supersonic stream. J. Spacecr. Rockets $1966,3: 1658-1665$

Schlatter P, Orlu R. Assessment of direct numerical simulation data of turbulent boundary layers. J.

Fluid. Mech., 2010, 659:116-126

Sun M B, Hu Z W. Formation of surface trailing counter-rotating vortex pairs downstream of a sonic jet in a supersonic crossflow. J. Fluid Mech. , 2018a, 850:551-583

Sun M B, Hu Z W. Generation of Upper Trailing Counter-Rotating Vortices of a Sonic Jet in a Supersonic Crossflow. AIAA J, 2018b, 56 (3):1047-1059

Sun M B, Hu Z W, Sandham N D. Recovery of a supersonic turbulent boundary layer after an expansion corner. Phys. Fluids, 2017, 29 (076103)

Sun M B, Zhang S P, Zhao Y H, Zhao Y X, Liang J H. Experimental investigation on transverse jet penetration into a supersonic turbulent crossflow. Sci. China Tech. Sci., 2013, 56 (8):1989-1998 Thompson K W. Time dependent boundary conditions for hyperbolic systems. J. Comput. Phys. , 1987, $68(1): 1-24$ 
Touber E. Unsteadiness in shock wave boundary layer interactions. $\mathrm{PhD}$ thesis, University of Southampton, 2010

Wang B, Sandham N D, Hu Z, W Liu. Numerical study of oblique shock-wave/boundary-layer interaction considering sidewall effect. J. Fluid Mech. , 2015, 767:526-561

Wang Q C, Wang Z G. Structural characteristics of the supersonic turbulent boundary layer subjected to concave curvature. Appl. Phy. Lett., 2016, 108:114102

Won S-H, Jeung I-S, Parent B, Choi J-Y. Numerical Investigation of Transverse Hydrogen Jet into Supersonic Crossflow Using Detached-Eddy Simulation. AIAA J., 2010, 48 (6):1047-1058

Xie Z T, Castro I P. Efficient generation of inflow conditions for large-eddy simulation of street-scale flows. Flow Turbul. Combust. , 2008, 81:449-470

Young A D. Boundary Layers - AIAA Education Series. Blackwell Science, 1989

Zhao Y X, Yi S H, Tian L F, Cheng Z Y. Supersonic flow imaging via nanoparticles. Sci. China Ser.

E-Tech. Sci., 2009, 52 (12):3640-3648 